\title{
NUFFT for the Efficient Spectral Domain MoM Analysis of a Wide Variety of Multilayered Periodic Structures
}

\author{
Miguel Camacho ${ }^{\circledR}$, Student Member, IEEE, Rafael R. Boix ${ }^{\circledR}$, Member, IEEE, \\ and Francisco Medina ${ }^{\mathbb{1}}$, Fellow, IEEE
}

\begin{abstract}
In this paper the Method of Moments (MoM) in the spectral domain is used for the analysis of multilayered structures containing periodic arrays of either patches or apertures. The patches and apertures may have many different geometries including complex surfaces limited by two parallel lines and two arbitrary curves, circular and elliptic rings, circular and elliptic arcs, and circular and elliptic sectors. Basis functions accounting for edge singularities are used in the approximation of the electric/magnetic current density on the patches/apertures, which enables a fast convergence of MoM with respect to the number of basis functions. Since the 2-D Fourier transforms of the basis functions cannot be obtained in closed-form, these Fourier transforms are efficiently computed by means of the nonuniform fast Fourier transform (NUFFT) algorithm. Results have been obtained for frequency-selective surfaces (FSSs), and for the elements used in the design of both reflectarray and metasurface antennas. The results obtained indicate that the software based on the NUFFT is only $15 \%$ slower than the standard spectral domain MoM software used for structures in which the 2-D Fourier transform of the basis functions is analytical, and between 50 and 80 times faster than CST.
\end{abstract}

Index Terms-Fourier transforms, moment of methods (MoM), multilayered media, periodic structures.

\section{INTRODUCTION}

$\mathbf{T}$ HE efficient analysis of multilayered periodic structures is of key importance for the design of microwave devices ranging from frequency-selective surfaces (FSSs) to reflectarray/transmitarray antennas and metasurface (MTS) leakywave antennas. FSSs paved the way by enabling the control of the transmission/reflection properties of very thin screens that

Manuscript received March 25, 2019; revised May 15, 2019; accepted May 26, 2019. Date of publication June 6, 2019; date of current version October 4, 2019. This work was supported in part by the Engineering and Physical Sciences Research Council (EPSRC), U.K., through the EPSRC Center for Doctoral Training in Metamaterials under Grant EP/L015331/1, in part by Junta de Andalucía under Project P12-TIC-1435, and in part by the Spanish Ministry of Science, Innovation and Universities with EU FEDER funds under Project CICYT TEC2016-75103-C2-1-R and Project CICYT TEC2017-84724-P. (Corresponding author: Rafael R. Boix.)

M. Camacho is with the Electromagnetic and Acoustic Materials Group, Department of Physics and Astronomy, University of Exeter, Exeter EX4 4 QL, U.K., and also with the School of Engineering and Applied Sciences, University of Pennsylvania, Philadelphia, PA 19104-6391 USA (e-mail:mc586@exeter.ac.uk).

R. R. Boix and F. Medina are with the Microwaves Group, Department of Electronics and Electromagnetism, College of Physics, University of Seville, 41012 Seville, Spain (e-mail: boix@us.es; medina@us.es).

Color versions of one or more of the figures in this article are available online at http://ieeexplore.iee.org.

Digital Object Identifier 10.1109/TAP.2019.2920361 work as bandpass or band-reject filters when made of apertures or patches respectively [1], [2]. Although these structures were strictly periodic, researchers were able to extend the methods developed for the analysis of FSSs to the analysis of nonperiodic structures with a spatially smooth variation, thanks to the use of the local periodicity assumption (LPA). One example is the design of reflectarray antennas, which are made of periodic arrangements of printed patches of different dimensions on a grounded dielectric, usually illuminated by a horn antenna [3]. By optimizing the geometry of each element in the antenna, one can compensate for the phase difference encountered between the waves scattered by the different elements. If the geometry of the elements varies slowly along the antenna, then the LPA allows for the analysis of each element as if it was surrounded by a periodic environment [3]. The LPA was validated experimentally, and was an enabling breakthrough for the fast and accurate design of reflectarray antennas [4]. More recently, the LPA has also been applied for the design of printed patch holographic or MTS antennas, based on the leaky-wave phenomena [5], [6]. These antennas are made of very sub-wavelength printed patches on a thin grounded substrate, which are treated through equivalent surface impedance concepts [7]. The very-subwavelength patterning of the surface also allows for a reliable use of the LPA. When the equivalent surface impedance is properly modulated along the surface of the antenna, one can achieve a largely controllable conversion between surface and space waves [5]-[7], whose radiation can be spatially-tailored to obtain the desired radiation diagram.

One of the numerical methods most frequently used for the analysis of multilayered periodic structures is the method of moments (MoM) in the spectral domain, which was introduced by Mittra and his coworkers [2]. The spectral domain MoM is very efficient provided that the 2-D Fourier transform of the basis functions used to approximate the magnetic current density on the apertures, or the electric current density on the patches, can be obtained in closed form. Two types of basis functions have been customarily used in the application of the spectral domain MoM: subsectional basis functions (such as rooftops, piecewise sinusoidal, etc.) and entire domain basis functions. Subsectional basis functions have the capability to be adaptable to a large variety of geometries, but their main drawback is that they lead to large MoM matrices to be inverted. Chan and Mittra [8] developed a fast Fourier 
transform (FFT) approach for the efficient computation of the spectral domain MoM matrix entries when subsectional basis functions are used, but this approach is restricted to cases where the patches or apertures fit into a uniform rectangular grid limited by the contour of the periodic unit cell. Entire domain basis functions accounting for the edge singularity condition are specially suitable for the application of MoM to multilayered periodic structures because they ensure a fast convergence of MoM with respect to the number of basis functions, and therefore, lead to small MoM matrices that can be easily inverted [9]-[11]. The nice properties of the edge singularity basis functions are due to the fact that they constitute a complete orthonormal set for the kernel of the integral equations to be solved as pointed out by Lerer and Schuchinsky [12]. Edge singularity basis functions have been proposed for simple canonical geometries such as rectangles [10], [12], parallelograms [12], circles [7], [13] and ellipses [7]. However, they have also been extended to more sophisticated shapes such as rings [14], split rings [11] or even more general surfaces limited by two parallel lines and two arbitrary curves [15]. The problem is that the 2-D Fourier transform of the edge singularity basis functions can be only obtained for the simplest geometries (rectangles, parallelograms, circles, and ellipses) [12], which prevents the use of these basis functions in the spectral domain MoM analysis of periodic structures with more complex shapes unless sophisticated mathematical tricks are used for each particular shape [11], [14].

In this paper the authors present a novel implementation of the spectral domain MoM for the analysis of multilayered periodic structures in which the Fourier transforms of the basis functions are numerically computed by means of the nonuniform fast Fourier transform (NUFFT) algorithm [16], [17]. This novel implementation has the relevant advantage that it can deal with edge singularity basis functions for which the 2-D Fourier transforms of the basis functions cannot be obtained in closed-form, and therefore, it enables the efficient MoM analysis of a large class of multilayered periodic structures. Also, the analysis of all these structures is carried out within a CPU time which is only slightly larger than that required in the cases where the 2-D Fourier transforms of the basis functions are available in closed-form. In previous papers, specially tailored mathematical tricks (interpolations in terms of Chebyshev polynomials, truncation of infinite series based on the properties of Bessel functions, etc.) have been used for the spectral domain MoM analysis of some particular periodic structures with edge-singularity basis functions for which 2-D Fourier transforms are not available in closed-form [11], [14]. The uniqueness of the spectral domain MoM with NUFFT as proposed in this paper is that this latter approach can be efficiently applied to a wide variety of different geometries with similar success. In particular, in this paper the new approach is applied to the analysis of periodic structures where the patches/apertures are surfaces limited by two parallel lines and two arbitrary curves [15], and to the cases where the patches/apertures are circular or elliptic rings [14], circular or elliptic arcs [11], and circular or elliptic sectors. The use of the NUFFT has made it possible to cover all these different cases since it does not require an equispaced grid for the computation of the samples used in the determination of the Fourier transform. This is in contrast to the standard FFT, which requires an equispaced uniform grid for the computation of the samples, and therefore, is more limited in terms of the number of different geometries that can be handled [8]. In this paper the spectral domain MoM with NUFFT has been applied to the determination of the transmission properties of FSSs made of apertures, to the determination of the phase curves of periodic structures used in the design of linearly and circularly polarized reflectarray antennas under the LPA, and finally, to the determination of the iso-frequency dispersion curves and the equivalent surface reactance of patches used in MTS antennas. The results obtained have been compared with previously published results and with results provided by the commercial software CST, and good agreement has been found in all cases. Our spectral domain MoM with NUFFT software has proven to be between 50 and 80 times faster than CST.

\section{NUMERICAL PROCEDURE}

Fig. 1(a) and (b) show a periodic array of patches printed on a two-layered substrate backed by a conducting plane. Similarly, Fig. 1(c) and (d) show a periodic array of apertures in a conducting screen, embedded in a two-layered substrate. The patches, the backing conducting plane and the conducting screen containing the apertures are assumed to be perfect electric conductors (PECs) of negligible thickness. Each of the two layers of the substrate of both periodic structures has a thickness $h_{i}(i=1,2)$, a complex permittivity $\varepsilon_{i}=\varepsilon_{0} \varepsilon_{r, i}\left(1-\mathrm{j} \tan \delta_{i}\right)(i=1,2)$, and a permeability $\mu_{0}$. Note that although the results presented in this paper are for periodic structures on one- or two-layered substrates, the derivations carried out in the rest of this Section for the structures of Fig. 1(a)-(d) can be easily extended to deal with general substrates containing an arbitrary number of layers. This only requires one to use adequate spectral domain dyadic Green's functions, which can be obtained for generic multilayered substrates by means of the recurrent algorithm described in [18]. Also, while the periodic structure of Fig. 1(c) and (d) acts as a bandpass FSS, the structure of Fig. 1(a) and (b) cannot be used as a reject band FSS unless the conducting plane is substituted by an interface with a semi-infinite region of free space [1], [2]. This latter possibility can also be easily contemplated in the formulation provided below by simply changing the spectral domain dyadic Green's function of the problem.

We will assume that the periodic unit cell of the periodic structures of Fig. 1(a)-(d) may have either $N_{e}$ patches $\left[N_{e}=2\right.$ in Fig. 1(b)] or $N_{e}$ apertures $\left[N_{e}=2\right.$ in Fig. 1(d)]. The possible shapes for these patches and apertures are shown in Fig. 2(a)-(g), and include surfaces limited by two parallel lines and two arbitrary curves (these surfaces encompass many different types of polygons, the barrel shape, the diabolo shape, etc.) of the type shown in Fig. 2(a), circular and elliptic rings [Fig. 2(b) and (c)], circular and elliptic arcs [Fig. 2(d) and (e)], and circular and elliptic sectors [Fig. 2(f) and (g)].

A plane wave is assumed to obliquely impinge on the two periodic structures of Fig. 1(a)-(d), its incidence direction 


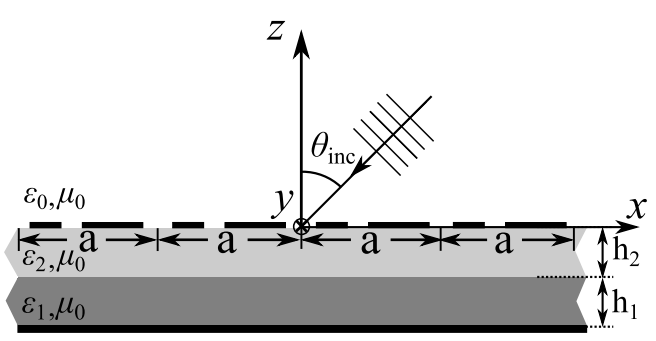

(a)

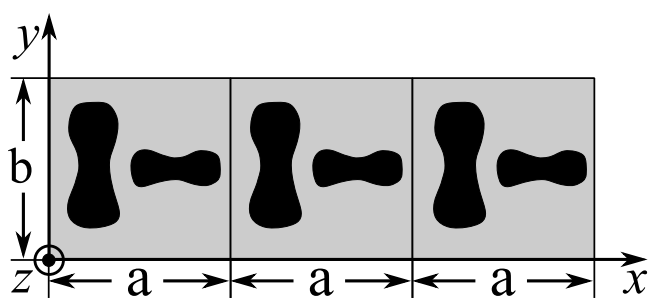

(b)

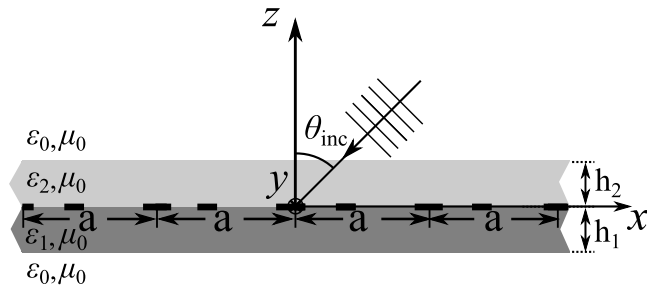

(c)

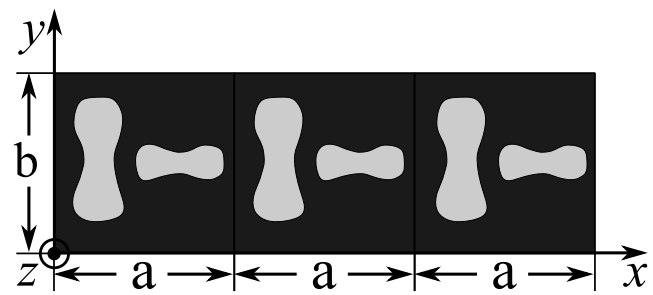

(d)

Fig. 1. (a) and (b) Side and top views of a periodic array of patches printed on a two-layered substrate backed by a conducting plane, respectively. (c) and (d) Side and top views of a periodic array of apertures embedded in a two-layered substrate, respectively. In both cases, a plane wave impinges on the periodic structures.

being given by the angular spherical coordinates $\theta_{\text {inc }}$ and $\varphi_{\text {inc }}$. In the following, a time dependence of the type $\mathrm{e}^{\mathrm{j} \omega t}$ will be assumed and suppressed throughout.

\section{A. MoM in the Spectral Domain}

In this subsection we describe the spectral domain MoM solution of the scattering problems posed in Fig. 1(a)-(d). Also, we briefly indicate how the solution of these scattering problems is used in the design of FSSs, reflectarray antennas and MTS antennas.

In order to determine the fields scattered by the periodic structure of Fig. 1(a) and (b), we need to determine the surface electric current density excited on the patches of the metallized interface $z=0, \mathbf{J}(x, y)$, by the impinging plane wave. This electric current density is the solution of the following electric

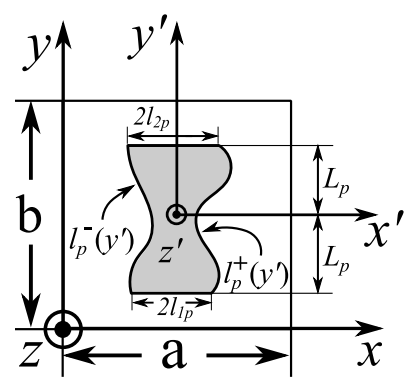

(a)

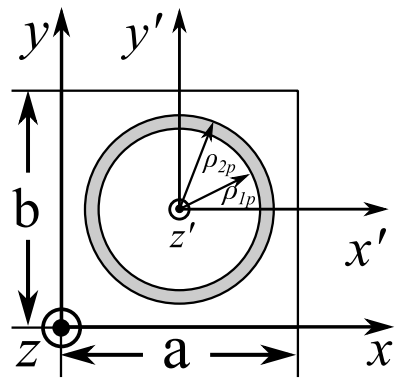

(b)

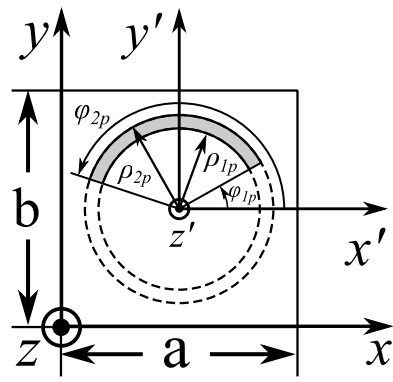

(d)

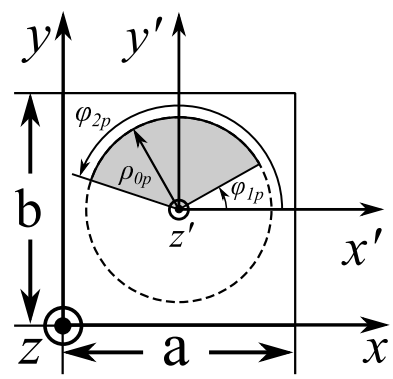

(f)

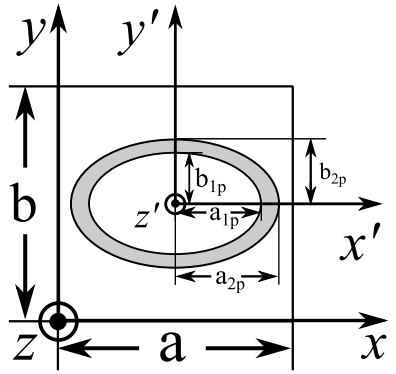

(c)

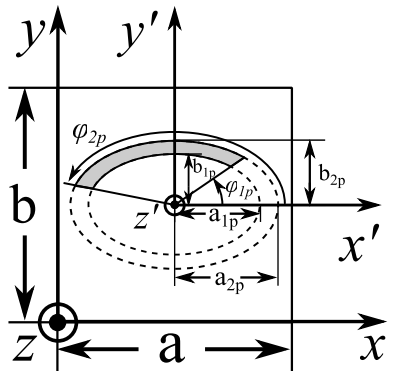

(e)

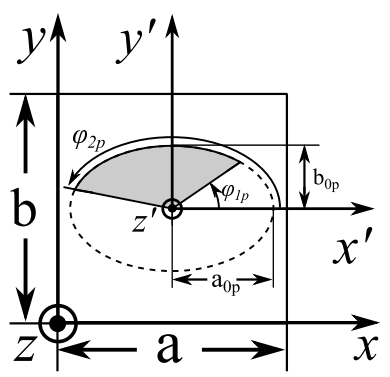

(g)
Fig. 2. Patches or apertures appearing in the unit cells of the periodic structures of Fig. 1. (a) Surface limited by two lines parallel to the $x^{\prime}$-axis, and by two arbitrary curves $x^{\prime}=l_{p}^{-}\left(y^{\prime}\right)$ and $x^{\prime}=l_{p}^{+}\left(y^{\prime}\right)$. (b) Circular and (c) elliptic rings. (d) Circular and (e) elliptic arcs. (f) Circular and (g) elliptic sectors.

field integral equation (EFIE):

$$
\begin{aligned}
\hat{\mathbf{z}} \times\left[\mathbf{E}^{\mathrm{ap}}(x, y, z=0)+\right. & \sum_{m=-\infty}^{+\infty} \sum_{n=-\infty}^{+\infty} \int_{P_{m n}} \bar{G}_{\mathrm{p}}^{E J} \\
\left(x-x^{\prime}, y-y^{\prime}, z\right. & \left.\left.=0, z^{\prime}=0\right) \cdot \mathbf{J}\left(x^{\prime}, y^{\prime}\right) d x^{\prime} d y^{\prime}\right]=\mathbf{0} \\
(x, y) \in P_{00} & \text { (1) }
\end{aligned}
$$

where $P_{m n}(m, n=\ldots,-1,0,1, \ldots)$ is the metallized portion of the $z=0$ plane within the $m n$-th periodic unit cell, 
$\mathbf{E}^{\mathrm{ap}}(x, y, z)$ is the electric field that would be generated in all space by the plane wave impinging on the multilayered structure of Fig. 1(a) and (b) in the absence of the patches, and $\overline{\mathbf{G}}_{\mathrm{p}}^{E J}\left(x-x^{\prime}, y-y^{\prime}, z=0, z^{\prime}=0\right)$ is a $2 \times 2$ matrix representing the dyadic Green's function that links the transverse (to $z$ ) electric field at the plane $z=0, \mathbf{E}_{t}(x, y, z=0)$, with the electric current density $\mathbf{J}\left(x^{\prime}, y^{\prime}\right)$ that originates this electric field.

Also, in order to determine the fields scattered by the periodic structure of Fig. 1(c) and (d), we need to determine the magnetic current density excited on the apertures of the conducting screen located at $z=0, \mathbf{M}(x, y)$, by the impinging plane wave. This magnetic current density is the solution of the following integral equation:

$$
\begin{array}{r}
\mathbf{J}^{\mathrm{aa}}(x, y)+\sum_{m=-\infty}^{+\infty} \sum_{n=-\infty}^{+\infty} \int_{A_{m n}} \\
\times \overline{\mathbf{G}}_{\mathrm{a}}^{J M}\left(x-x^{\prime}, y-y^{\prime}, z=0, z^{\prime}=0\right) \cdot \mathbf{M}\left(x^{\prime}, y^{\prime}\right) d x^{\prime} d y^{\prime}=\mathbf{0} \\
\quad(x, y) \in A_{00}
\end{array}
$$

where $A_{m n}$ is the set of apertures existing in the $z=0$ plane within the $m n$-th unit cell, $\mathbf{J}^{\mathrm{aa}}(x, y)$ is the surface electric current density that would be induced at the conducting plane $z=0$ of Fig. 1(c) and (d) by the impinging wave if the apertures were not present, and $\overline{\mathbf{G}}_{\mathrm{a}}^{J M}\left(x-x^{\prime}, y-y^{\prime}, z=0, z^{\prime}=0\right)$ is a $2 \times 2$ matrix representing the dyadic Green's function that links the surface electric current density (discontinuity in the tangential magnetic field) existing at the $z=0$ plane with the magnetic current density $\mathbf{M}\left(x^{\prime}, y^{\prime}\right)$ that originates this electric current density.

Since $\mathbf{J}(x, y)$ and $\mathbf{M}(x, y)$ are Floquet-periodic functions of $x$ and $y$, in order to solve the integral equations of (1) and (2), we only need to determine $\mathbf{J}(x, y)$ and $\mathbf{M}(x, y)$ within one periodic unit cell, e.g., the domain $C_{00}=\{0 \leq x \leq a$; $0 \leq$ $y \leq b\}$. Looking for a solution of the integral equations, we expand $\mathbf{J}(x, y)$ and $\mathbf{M}(x, y)$ in $P_{00}$ and $A_{00}$ respectively in terms of known basis functions as follows:

$$
\begin{aligned}
\mathbf{J}(x, y) & =\sum_{p=1}^{N_{\mathrm{e}}} \sum_{q=1}^{2} \sum_{r=1}^{N_{1 q}} \sum_{s=1}^{N_{2 q}} c_{r s}^{p q} \mathbf{J}_{r s}^{p q}(x, y) \\
\mathbf{M}(x, y) & =\sum_{p=1}^{N_{\mathrm{e}}} \sum_{q=1}^{2} \sum_{r=1}^{N_{1 q}} \sum_{s=1}^{N_{2 q}} d_{r s}^{p q} \mathbf{M}_{r s}^{p q}(x, y) .
\end{aligned}
$$

In (3)/(4) the superindex $p\left(p=1, \ldots, N_{\mathrm{e}}\right)$ indicates the number of the patch/aperture in $P_{00} / A_{00}$ for a total of $N_{\mathrm{e}}$ patches/apertures in Fig. 1(b) and (d). The superindex $q(q=1,2)$ indicates the component of $\mathbf{J}(x, y) / \mathbf{M}(x, y)$ which is being approximated by the basis function $\mathbf{J}_{r s}^{p q}(x, y) / \mathbf{M}_{r s}^{p q}(x, y)$. And since $\mathbf{J}(x, y) / \mathbf{M}(x, y)$ depends on two coordinates (which can be cartesian coordinates, polar coordinates [7], [11], [14] or stretched polar coordinates suitable for patches and apertures with elliptical shape [7], [12]), the subindex $r\left(r=1, \ldots, N_{1 q}\right)$ is swept to fit the dependence of $\mathbf{J}(x, y) / \mathbf{M}(x, y)$ on one of the coordinates, while the subindex $s\left(s=1, \ldots, N_{2 q}\right)$ is swept to fit the dependence of $\mathbf{J}(x, y) / \mathbf{M}(x, y)$ on the other coordinate. Thus, the total number of basis functions per patch (aperture) turns out to be $N_{b}=N_{11} N_{21}+N_{12} N_{22}$.
When (3) and (4) are substituted in (1) and (2), and Galerkin's version of MoM is applied, the following systems of equations are obtained for the unknown coefficients $c_{r s}^{p q}$ and $d_{r s}^{p q}$ of (3) and (4)

$$
\begin{aligned}
& \sum_{p=1}^{N_{\mathrm{e}}} \sum_{q=1}^{2} \sum_{r=1}^{N_{1 q}} \sum_{s=1}^{N_{2 q}} \Gamma_{k l, r s}^{i j, p q} c_{r s}^{p q}=e_{k l}^{i j} \\
& \left(i=1, \ldots, N_{\mathrm{e}} ; j=1,2 ; k=1, \ldots, N_{1 j} ; l=1, \ldots, N_{2 j}\right) \\
& \sum_{p=1}^{N_{\mathrm{e}}} \sum_{q=1}^{2} \sum_{r=1}^{N_{1 q}} \sum_{s=1}^{N_{2 q}} \Lambda_{k l, r s}^{i j, p q} d_{r s}^{p q}=f_{k l}^{i j} \\
& \left(i=1, \ldots, N_{\mathrm{e}} ; j=1,2 ; k=1, \ldots, N_{1 j} ; l=1, \ldots, N_{2 j}\right) .
\end{aligned}
$$

If we invoke Parseval's identity for 2-D Fourier transforms, the MoM matrix entries $\Gamma_{k l, r s}^{i j, p q}$ and $\Lambda_{k l, r s}^{i j, p q}$ of (5) and (6) can be expressed in the spectral domain as double infinite summations given by [2]

$$
\begin{aligned}
& \Gamma_{k l, r s}^{i j, p q} \\
& =a b \sum_{m=-\infty}^{+\infty} \sum_{n=-\infty}^{+\infty}\left[\left(\widetilde{\mathbf{J}}_{k l}^{i j, \mathrm{~d}}\left(k_{x m}, k_{y n}\right)\right)^{*}\right]^{t} \\
& \quad \cdot \widetilde{\mathbf{G}}_{\mathrm{p}}^{E J, \mathrm{c}}\left(k_{x}=k_{x m}, k_{y}=k_{y n}, z=0, z^{\prime}=0\right) \cdot \widetilde{\mathbf{J}}_{r s}^{p q, \mathrm{~d}}\left(k_{x m}, k_{y n}\right)
\end{aligned}
$$

$\Lambda_{k l, r s}^{i j, p q}$

$$
\begin{aligned}
= & a b \sum_{m=-\infty}^{+\infty} \sum_{n=-\infty}^{+\infty}\left[\left(\widetilde{\mathbf{M}}_{k l}^{i j, \mathrm{~d}}\left(k_{x m}, k_{y n}\right)\right)^{*}\right]^{t} \\
& \cdot \widetilde{\widetilde{\mathbf{G}}}_{\mathrm{a}}^{J M, \mathrm{c}}\left(k_{x}=k_{x m}, k_{y}=k_{y n}, z=0, z^{\prime}=0\right) \cdot \widetilde{\mathbf{M}}_{r s}^{p q, \mathrm{~d}}\left(k_{x m}, k_{y n}\right)
\end{aligned}
$$

where $k_{x m}=k_{0} \sin \theta_{\text {inc }} \cos \varphi_{\text {inc }}+2 \pi m / a\left(k_{0}=\omega \sqrt{\varepsilon_{0} \mu_{0}}=\right.$ $\left.2 \pi / \lambda_{0}\right)$ and $k_{y n}=k_{0} \sin \theta_{\text {inc }} \sin \varphi_{\text {inc }}+2 \pi n / b$.

In (7) and (8) the $2 \times 2$ matrices $\widetilde{\mathbf{G}}_{\mathrm{p}}^{E J, \mathrm{c}}\left(k_{x}, k_{y}, z=0, z^{\prime}=\right.$ $0)$ and $\widetilde{\overline{\mathbf{G}}}_{\mathrm{a}}^{J M, \mathrm{c}}\left(k_{x}, k_{y}, z=0, z^{\prime}=0\right)$ stand for the continuous 2-D Fourier transforms of $\overline{\mathbf{G}}_{\mathrm{p}}^{E J}\left(x, y, z=0, z^{\prime}=0\right)$ and $\overline{\mathbf{G}}_{\mathrm{a}}^{J M}\left(x, y, z=0, z^{\prime}=0\right)$. It can be easily shown that

$$
\begin{aligned}
\widetilde{\mathbf{G}}_{\mathrm{a}}^{J M, \mathrm{c}}\left(k_{x}, k_{y}, z=0, z^{\prime}=0\right) \\
\quad=\left[\widetilde{\mathbf{G}}_{\mathrm{a}}^{E J, \mathrm{c}}\left(k_{x}, k_{y}, z=0, z^{\prime}=0\right)\right]^{-1} \cdot\left(\begin{array}{cc}
0 & -1 \\
1 & 0
\end{array}\right)
\end{aligned}
$$

where $\widetilde{\overline{\mathbf{G}}}_{\mathrm{a}}^{E J, \mathrm{c}}\left(k_{x}, k_{y}, z=0, z^{\prime}=0\right)$ is the continuous 2-D Fourier transform of the dyadic Green's function relating the transverse (to $z$ ) electric field and the electric current density at the $z=0$ plane of Fig. 1(c). The matrix $\widetilde{\mathbf{G}}_{\mathrm{a}}^{E J, \mathrm{c}}\left(k_{x}, k_{y}, z=0, z^{\prime}=0\right)$ is not to be confused with $\widetilde{\mathbf{G}}_{\mathrm{p}}^{E J, \mathrm{c}}\left(k_{x}, k_{y}, z=0, z^{\prime}=0\right)$ since $z=0$ in Fig. $1(\mathrm{c})$ is in between the two layers and the two-layered medium is limited by two semi-infinite half-spaces up and down, while $z=0$ in Fig. 1(a) is above the two layers and the two-layered medium is limited by one semi-infinite halfspace up and by a conducting plane down. Both matrices $\widetilde{\overline{\mathbf{G}}}_{\mathrm{a}}^{E J, \mathrm{c}}\left(k_{x}, k_{y}, z=0, z^{\prime}=0\right)$ and $\widetilde{\mathbf{G}}_{\mathrm{p}}^{E J, \mathrm{c}}\left(k_{x}, k_{y}, z=0, z^{\prime}=0\right)$ can be obtained in closed-form as explained in [18]. 
The spectral domain basis functions $\widetilde{\mathbf{J}}_{r s}^{p q, \mathrm{~d}}\left(k_{x m}, k_{y n}\right)$ and $\widetilde{\mathbf{M}}_{r s}^{p q, \mathrm{~d}}\left(k_{x m}, k_{y n}\right)\left(p=1, \ldots, N_{\mathrm{e}} ; q=1,2 ; r=\right.$ $\left.1, \ldots, N_{1 q} ; s=1, \ldots, N_{2 q}\right)$ of (7) and (8) stand for the discrete 2-D Fourier transforms of $\mathbf{J}_{r s}^{p q}(x, y)$ and $\mathbf{M}_{r s}^{p q}(x, y)$ respectively, and are given by

$$
\begin{aligned}
\widetilde{\mathbf{J}}_{r s}^{p q, \mathrm{~d}}\left(k_{x m}, k_{y n}\right) & =\frac{1}{a b} \int_{P_{00}} \mathbf{J}_{r s}^{p q}(x, y) \mathrm{e}^{-\mathrm{j}\left(k_{x m} x+k_{y n} y\right)} d x d y \\
\widetilde{\mathbf{M}}_{r s}^{p q, \mathrm{~d}}\left(k_{x m}, k_{y n}\right) & =\frac{1}{a b} \int_{A_{00}} \mathbf{M}_{r s}^{p q}(x, y) \mathrm{e}^{-\mathrm{j}\left(k_{x m} x+k_{y n} y\right)} d x d y .
\end{aligned}
$$

Finally, the coefficients $e_{k l}^{i j}$ and $f_{k l}^{i j}$ of the two systems of equations (5) and (6) can be obtained in the spectral domain as

$$
\begin{aligned}
& e_{k l}^{i j}=-a b[ {\left.\left[\widetilde{\mathbf{J}}_{k l}^{i j, \mathrm{~d}}\left(k_{x 0}, k_{y 0}\right)\right)^{*}\right]^{t} \cdot \mathbf{E}^{\mathrm{ap}}(x, y, z=0) } \\
& \times \mathrm{e}^{-\mathrm{j} k_{0}\left(\sin \theta_{\mathrm{inc}} \cos \varphi_{\text {inc }} x+\sin \theta_{\text {inc }} \sin \varphi_{\text {inc }} y\right)} \\
& f_{k l}^{i j}=-a b\left[\left(\widetilde{\mathbf{M}}_{k l}^{i j, \mathrm{~d}}\left(k_{x 0}, k_{y 0}\right)\right)^{*}\right]^{t} \cdot \mathbf{J}^{\mathrm{aa}}(x, y) \\
& \times \mathrm{e}^{-\mathrm{j} k_{0}\left(\sin \theta_{\mathrm{inc}} \cos \varphi_{\text {inc }} x+\sin \theta_{\text {inc }} \sin \varphi_{\text {inc }} y\right)}
\end{aligned}
$$

where the factor $\mathrm{e}^{-\mathrm{j} k_{0}\left(\sin \theta_{\text {inc }} \cos \varphi_{\text {inc }} x+\sin \theta_{\text {inc }} \sin \varphi_{\text {inc }} y\right)}$ has been explicitly included in (12) and (13) to absorb the dependence of $\mathbf{E}^{\mathrm{ap}}(x, y, z=0)$ and $\mathbf{J}^{\mathrm{aa}}(x, y)$ on $x$ and $y$. Note that both $\mathbf{E}^{\mathrm{ap}}(x, y, z=0)$ and $\mathbf{J}^{\mathrm{aa}}(x, y)$ include a phase factor of the type $\mathrm{e}^{+\mathrm{j} k_{0}\left(\sin \theta_{\text {inc }} \cos \varphi_{\text {inc }} x+\sin \theta_{\text {inc }} \sin \varphi_{\text {inc }} y\right)}$ (nonvanishing for oblique incidence, i. e., $\theta_{\text {inc }} \neq 0$ ), which has to be removed in (12) and (13) by means of the annihilating phase factor $\mathrm{e}^{-\mathrm{j} k_{0}\left(\sin \theta_{\text {inc }} \cos \varphi_{\text {inc }} x+\sin \theta_{\text {inc }} \sin \varphi_{\text {inc }} y\right)}$ since the coefficients $e_{k l}^{i j}$ and $f_{k l}^{i j}$ are not dependent on $x$ and $y$.

As mentioned above, the periodic structure of Fig. 1(c) and (d) is a bandpass FSS. Once the integral equation (2) is solved by means of MoM and $\mathbf{M}(x, y)$ is obtained, the reflection and transmission coefficients of the $m n$-th Floquet harmonic of the FSS, $R_{m n}$ and $T_{m n}$, can be obtained in terms of $\mathbf{M}(x, y)$ as explained elsewhere (e. g., see [2, eqs. (75)-(78)] and [19, eqs. (24)-(27)]).

As commented in Section I of the paper, the design of reflectarray antennas is customarily carried out by means of the LPA. In the frame of the LPA, the phase shift in each reflectarray element is computed as if the element were surrounded by a periodic environment. In this sense, the configurations of Fig. 1(a) and (b) represent the typical periodic structures that are used for the characterization of the elements of a reflectarray. Let us assume that one of these periodic structures contains the element used in the design of a linearly polarized antenna, and let us assume that the value of the operating frequency does not allow the excitation of grating lobes when the plane wave impinges on the periodic structure (which implies that $\max (a, b)<\lambda_{0} /\left(1+\sin \theta_{\text {inc }}\right)$ [1]). Under these conditions, the reflectarray element surrounded by a periodic environment can be characterized by means of a $2 \times 2$ linear polarization reflection matrix, $\overline{\mathbf{R}}_{\mathrm{LP}}$, defined as [20]

$$
\left(\begin{array}{l}
E_{x}^{\mathrm{ref}} \\
E_{y}^{\mathrm{ref}}
\end{array}\right)=\overline{\mathbf{R}}_{\mathrm{LP}} \cdot\left(\begin{array}{l}
E_{x}^{\mathrm{inc}} \\
E_{y}^{\text {inc }}
\end{array}\right)=\left(\begin{array}{ll}
R_{x x} & R_{x y} \\
R_{y x} & R_{y y}
\end{array}\right) \cdot\left(\begin{array}{l}
E_{x}^{\mathrm{inc}} \\
E_{y}^{\text {inc }}
\end{array}\right)
$$

where $E_{x}^{\text {inc }}$ and $E_{y}^{\text {inc }}$ are the complex amplitudes of the transverse (to $z$ ) components of the electric field of the impinging plane wave, and $E_{x}^{\text {ref }}$ and $E_{y}^{\text {ref }}$ are the complex amplitudes of the transverse (to $z$ ) components of the electric field of the reflected $m=n=0$ Floquet harmonic (the only reflected propagating harmonic in the absence of grating lobes). Once the integral equation (1) is solved, the quantities $E_{x}^{\text {ref }}$ and $E_{y}^{\text {ref }}$ can be readily obtained in terms of $\mathbf{J}(x, y)$. In reflectarray antenna design, the phases of the matrix coefficients $R_{x x}$ and $R_{y y}$ of (14) are crucial for the determination of the element dimensions that provide the adequate phase shift for a prescribed radiation pattern, and the magnitudes of $R_{x y}$ and $R_{y x}$ are useful to give an estimation of the cross-polarization level introduced by the element.

If the reflectarray to be designed is a circularly polarized antenna, in the absence of grating lobes each reflectarray element has to be characterized by means of a $2 \times 2$ circular polarization reflection matrix, $\overline{\mathbf{R}}_{\mathrm{CP}}$, which can be defined as [11]

$$
\begin{aligned}
\left(\begin{array}{l}
E_{\mathrm{RHCP}}^{\mathrm{ref}} \\
E_{\mathrm{LHCP}}^{\mathrm{ref}}
\end{array}\right) & =\overline{\mathbf{R}}_{\mathrm{CP}} \cdot\left(\begin{array}{l}
E_{\mathrm{RHCP}}^{\mathrm{inc}} \\
E_{\mathrm{LHCP}}^{\text {nc }}
\end{array}\right) \\
& =\left(\begin{array}{ll}
R_{\mathrm{RHCP}, \mathrm{RHCP}} & R_{\mathrm{RHCP}, \mathrm{LHCP}} \\
R_{\mathrm{LHCP}, \mathrm{RHCP}} & R_{\mathrm{LHCP}, \mathrm{LHCP}}
\end{array}\right) \cdot\left(\begin{array}{l}
E_{\mathrm{RHCP}}^{\mathrm{inc}} \\
E_{\mathrm{LHCP}}^{\text {inc }}
\end{array}\right)
\end{aligned}
$$

where $E_{\mathrm{RHCP}}^{\mathrm{inc}}$ and $E_{\mathrm{LHCP}}^{\mathrm{inc}}$ are the complex amplitudes of the right-hand circular polarization (RHCP) and left-hand circular polarization (LHCP) components of the electric field of the impinging wave, and $E_{\mathrm{RHCP}}^{\mathrm{ref}}$ and $E_{\mathrm{LHCP}}^{\mathrm{ref}}$ are the RHCP and LHCP components of the electric field of the reflected $m=n=0$ Floquet harmonic. These latter components can be readily obtained in terms of $\mathbf{J}(x, y)$ once the integral equation (1) is solved. As in the case of linear polarization antennas, the phases of the matrix coefficients $R_{\text {RHCP,RHCP }}$ and $R_{\mathrm{LHCP}, \mathrm{LHCP}}$ of (15) are the key parameters that make it possible to adjust the dimensions of each reflectarray element to obtain the required phase shift.

The periodic structures of Fig. 1(a) and (c) may support bound (nonleaky) surface wave modes that are attenuated along the $z$-direction (for these modes, the phase constant is larger than $k_{0}$ ). In order to obtain the propagation constant, $k_{\rho \text { sw }}$, of the fundamental homogeneous Floquet mode $m=$ $n=0$ that propagates in a direction making an angle $\alpha$ with the positive $x$ axis, we have to redefine $k_{x m}$ and $k_{y n}$ in (7) and (8) as

$$
\begin{aligned}
& k_{\mathrm{xm}}=k_{\rho \mathrm{sw}} \cos \alpha+2 \pi m / a \\
& k_{\mathrm{yn}}=k_{\rho \mathrm{sw}} \sin \alpha+2 \pi n / b .
\end{aligned}
$$

With this definition of $k_{x m}$ and $k_{y n}$, the values of $k_{\rho \mathrm{sw}}$ for fixed values of $\alpha$ and $k_{0}$ (i.e., for fixed values of frequency) can be found as the solutions of the transcendental equations [7], [21]

$$
\begin{aligned}
& \operatorname{det}\left[\Gamma_{k l, r s}^{i j, p q}\left(k_{\rho \mathrm{sw}}, \alpha, k_{0}\right)\right]=0 \\
& \operatorname{det}\left[\Lambda_{k l, r s}^{i j, p q}\left(k_{\rho \mathrm{sw}}, \alpha, k_{0}\right)\right]=0
\end{aligned}
$$

where $\Gamma_{k l, r s}^{i j, p q}$ and $\Lambda_{k l, r s}^{i j, p q}$ are the MoM matrices (7) and (8) of the linear systems of equations (5) and (6) respectively.

In practical applications in which the periodic structure of Fig. 1(a) and (b) acts as an impedance surface or an 
MTS made of sub-wavelength elements [7], [22], focus is concentrated on the isofrequency dispersion curves of the fundamental Floquet mode $m=n=0$. In these dispersion curves, $k_{\rho \mathrm{sw}}=k_{\rho \mathrm{sw}}(\alpha)$ is represented in polar coordinates for fixed values of frequency [7], [22]. The dispersion curves also provide important information about the phase and group velocities of the surface wave under study.

The design of aperture MTS antennas is done through the concept of impenetrable equivalent surface reactance. By spatially modulating such reactance, one can achieve leakage from the aforementioned surface waves. The implementation of the optimal modulation requires the knowledge of the dependence of the reactance values on a pair of geometrical parameters of the element in the unit cell such as size, angle of rotation, eccentricity in the case of an elliptic element, etc. This dependence is usually represented as a colormap that provides the design curves from which one can extract the desired values of the modulated reactance [6], [7]. The equivalent impenetrable reactance of the MTS periodic structure is defined in terms of $k_{\rho \mathrm{sw}}$ as [7]

$$
X_{s}=\zeta_{0} \sqrt{\left(\frac{k_{\rho \mathrm{sw}}}{k_{0}}\right)^{2}-1}
$$

where $\zeta_{0}=\sqrt{\mu_{0} / \varepsilon_{0}}$ is the free-space impedance.

\section{B. Basis Functions for the Electric and Magnetic Current Densities}

The choice of basis functions for $\mathbf{J}(x, y) / \mathbf{M}(x, y)$ in the patches/apertures of Fig. 1(a)-(d) is crucial for an efficient implementation of the spectral domain MoM of Subsection II-A. As commented in Section I, the basis functions accounting for edge singularities ensure a fast convergence of MoM with respect to the number of basis functions, and therefore, lead to an efficient implementation of MoM since the size of the MoM matrices to be inverted (i.e., the coefficient matrices appearing in the linear systems of equations (5) an (6)) turns out to be small. In this subsection we define the edge singularity basis functions for all the geometries shown in Fig. 2(a)-(g). It can be verified that the components of the basis functions parallel to the edges of the patches/apertures become singular at the points of these edges. Also, the components of the basis functions that are normal to the edges of the patches/apertures become zero at the points of these edges, their derivative along the normal direction being singular at these points. These are physical constraints to be expected for the electric/magnetic current densities existing on the patches/apertures. The results presented in Section III will confirm that the edge singularity basis functions chosen for the geometries of Fig. 2(a)-(g) all lead to a fast convergence of MoM with respect to the number of these basis functions.

Let $\mathbf{B}_{r s}^{p q}(x, y)$ represent any of the two basis functions $\mathbf{J}_{r s}^{p q}(x, y)$ and $\mathbf{M}_{r s}^{p q}(x, y)$. Let $\left\{x^{\prime}, y^{\prime}, z^{\prime}\right\}$ be a shifted system of coordinates centered at the point $\left(x=x_{\mathrm{oc}}, y=y_{\mathrm{oc}}, z=0\right)$ of the domain $C_{00}$ of Fig. 1(b) and (d) [see the new system of coordinates $\left\{x^{\prime}, y^{\prime}, z^{\prime}\right\}$ in Fig. 2(a)-(g) $]$. The relation between the new cartesian coordinates and the original cartesian coordinates of Fig. 1(b) and (d) is given by

$$
\begin{aligned}
& x^{\prime}=x-x_{\mathrm{oc}} \\
& y^{\prime}=y-y_{\mathrm{oc}} \\
& z^{\prime}=z .
\end{aligned}
$$

For patches and apertures which fit the geometrical shape shown in Fig. 2(a), we propose to use the following edge singularity basis functions introduced in [15]

$$
\begin{aligned}
& \mathbf{B}_{r s}^{p 1}\left(x^{\prime}, y^{\prime}\right) \\
& =\frac{1}{l_{p}\left(y^{\prime}\right)} \frac{T_{r-1}\left(\frac{x^{\prime}-a_{p}\left(y^{\prime}\right)}{l_{p}\left(y^{\prime}\right)}\right)}{\sqrt{1-\left(\frac{x^{\prime}-a_{p}\left(y^{\prime}\right)}{l_{p}\left(y^{\prime}\right)}\right)^{2}}} U_{s-1}\left(\frac{y^{\prime}}{L_{p}}\right) \sqrt{1-\left(\frac{y^{\prime}}{L_{p}}\right)^{2}} \\
& \quad \times\left\{\left[\frac{x^{\prime}-a_{p}\left(y^{\prime}\right)}{l_{p}\left(y^{\prime}\right)} \frac{d l_{p}\left(y^{\prime}\right)}{d y^{\prime}}+\frac{d a_{p}\left(y^{\prime}\right)}{d y^{\prime}}\right] \hat{\mathbf{x}}+\hat{\mathbf{y}}\right\} \\
& \quad\left(l_{p}^{-}\left(y^{\prime}\right)<x^{\prime}<l_{p}^{+}\left(y^{\prime}\right) ;-L_{p} \leq y^{\prime} \leq+L_{p}\right) \\
& \quad\left(r=1, \ldots, N_{11} ; s=1, \ldots, N_{21}\right) \\
& =\frac{1}{L_{p}} U_{r-1}^{p 2}\left(\frac{x^{\prime}-a_{p}\left(y^{\prime}\right)}{l_{p}\left(y^{\prime}\right)}\right) \sqrt{1-\left(\frac{x^{\prime}-a_{p}\left(y^{\prime}\right)}{l_{p}\left(y^{\prime}\right)}\right)^{2}} \\
& \quad \times \frac{T_{s-1}\left(\frac{y^{\prime}}{L_{p}}\right)}{\sqrt{1-\left(\frac{y^{\prime}}{L_{p}}\right)^{2}}} \hat{\mathbf{x}} \quad\left(l_{p}^{-}\left(y^{\prime}\right) \leq x^{\prime} \leq l_{p}^{+}\left(y^{\prime}\right) ;-L_{p}<y^{\prime}<+L_{p}\right) \\
& \left(r=1, \ldots, N_{12} ; s=1, \ldots, N_{22}\right)
\end{aligned}
$$

where $T_{r-1}(\cdot)\left(T_{s-1}(\cdot)\right)$ and $U_{r-1}(\cdot)\left(U_{s-1}(\cdot)\right)$ are Chebyshev polynomials of first and second kind respectively. The functions $a_{p}\left(y^{\prime}\right)$ and $l_{p}\left(y^{\prime}\right)$ of (24) and (25) are given by

$$
\begin{aligned}
& a_{p}\left(y^{\prime}\right)=\frac{l_{p}^{+}\left(y^{\prime}\right)+l_{p}^{-}\left(y^{\prime}\right)}{2} \\
& l_{p}\left(y^{\prime}\right)=\frac{l_{p}^{+}\left(y^{\prime}\right)-l_{p}^{-}\left(y^{\prime}\right)}{2}
\end{aligned}
$$

where $x^{\prime}$ and $y^{\prime}$ can be obtained in terms of $x$ and $y$ as shown in (21) and (22), and where $x=l_{p}^{-}\left(y^{\prime}\right)$ and $x=l_{p}^{+}\left(y^{\prime}\right)$ $\left(-L_{p} \leq y^{\prime} \leq+L_{p}\right)$ are the equations of the two curves that limit the left side and the right side of the patch/aperture of Fig. 2(a).

Let us now introduce polar coordinates $\rho^{\prime}$ and $\varphi^{\prime}$ for the shifted system of coordinates $\left\{x^{\prime}, y^{\prime}, z^{\prime}\right\}$. According to (21) and (22), these coordinates are related to the cartesian coordinates of Fig. 1(b) and (d) by means of

$$
\begin{aligned}
& \rho^{\prime} \cos \varphi^{\prime}=x-x_{\mathrm{oc}} \\
& \rho^{\prime} \sin \varphi^{\prime}=y-y_{\mathrm{oc}} .
\end{aligned}
$$


For the circular ring of Fig. 2(b), we propose the use of the edge singularity basis functions [14], [23]

$$
\begin{aligned}
\mathbf{B}_{r s}^{p 1}\left(\rho^{\prime}, \varphi^{\prime}\right) & \\
= & \frac{T_{r-1}\left(\frac{2}{\rho_{2 p}-\rho_{1 p}}\left[\rho^{\prime}-\frac{\rho_{2 p}+\rho_{1 p}}{2}\right]\right)}{\sqrt{1-\left(\frac{2}{\rho_{2 p}-\rho_{1 p}}\left[\rho^{\prime}-\frac{\rho_{2 p}+\rho_{1 p}}{2}\right]\right)^{2}}} \\
& \times \mathrm{e}^{\mathrm{j}\left[s-\left(\frac{N_{21}+1}{2}\right)\right] \varphi^{\prime}\left(-\sin \varphi^{\prime} \hat{\mathbf{x}}+\cos \varphi^{\prime} \hat{\mathbf{y}}\right)} \\
& \left(\rho_{1 p}<\rho^{\prime}<\rho_{2 p} ; 0 \leq \varphi^{\prime}<2 \pi\right) \\
& \left(r=1, \ldots, N_{11} ; s=1, \ldots, N_{21} ; N_{21} \text { odd }\right) \\
\mathbf{B}_{r s}^{p 2} & \left(\rho^{\prime}, \varphi^{\prime}\right) \\
= & U_{r-1}\left(\frac{2}{\rho_{2 p}-\rho_{1 p}}\left[\rho^{\prime}-\frac{\rho_{2 p}+\rho_{1 p}}{2}\right]\right) \\
\quad & \quad \sqrt{1-\left(\frac{2}{\rho_{2 p}-\rho_{1 p}}\left[\rho^{\prime}-\frac{\rho_{2 p}+\rho_{1 p}}{2}\right]\right)^{2}} \mathrm{e}^{\mathrm{j}\left[s-\left(\frac{N_{22}+1}{2}\right)\right] \varphi^{\prime}} \\
\quad & \left(\cos \varphi^{\prime} \hat{\mathbf{x}}+\sin \varphi^{\prime} \hat{\mathbf{y}}\right) \quad\left(\rho_{1 p} \leq \rho^{\prime} \leq \rho_{2 p} ; 0 \leq \varphi^{\prime}<2 \pi\right) \\
& \left(r=1, \ldots, N_{12} ; s=1, \ldots, N_{22} ; N_{22} \text { odd }\right) .
\end{aligned}
$$

For the case of the elliptical ring of Fig. 2(c), we will assume that $b_{2 p} / a_{2 p}=b_{1 p} / a_{1 p}=\eta<1$ (i.e., we will assume that the axial ratios between the minor semi-axis and the major semi-axis are the same for the two ellipses limiting the rings). Under this assumption, we are going to define stretched polar coordinates $\alpha^{\prime}$ and $\beta^{\prime}$ for the shifted system of coordinates $\left\{x^{\prime}, y^{\prime}, z^{\prime}\right\}$, which are related to the coordinates $x$ and $y$ of Fig. 1(b) and (d) by means of

$$
\begin{aligned}
\alpha^{\prime} \cos \beta^{\prime} & =x-x_{\mathrm{oc}} \\
\eta \alpha^{\prime} \sin \beta^{\prime} & =y-y_{\mathrm{oc}} .
\end{aligned}
$$

The edge singularity basis functions $\mathbf{B}_{r s}^{p q}$ chosen for the elliptical ring of Fig. 2(c) can be written in terms of $\alpha^{\prime}$ and $\beta^{\prime}$ as

$$
\begin{aligned}
\mathbf{B}_{r s}^{p 1} & \left(\alpha^{\prime}, \beta^{\prime}\right) \\
= & \frac{T_{r-1}\left(\frac{2}{a_{2 p}-a_{1 p}}\left[\alpha^{\prime}-\frac{a_{2 p}+a_{1 p}}{2}\right]\right)}{\sqrt{1-\left(\frac{2}{a_{2 p}-a_{1 p}}\left[\alpha^{\prime}-\frac{a_{2 p}+a_{1 p}}{2}\right]\right)^{2}}} \\
& \times \mathrm{e}^{\mathrm{j}\left[s-\left(\frac{N_{21}+1}{2}\right)\right] \beta^{\prime}\left(-\sin \beta^{\prime} \hat{\mathbf{x}}+\eta \cos \beta^{\prime} \hat{\mathbf{y}}\right)} \\
& \left(a_{1 p}<\alpha^{\prime}<a_{2 p} ; 0 \leq \beta^{\prime}<2 \pi\right) \\
& \left(r=1, \ldots, N_{11} ; s=1, \ldots, N_{21} ; N_{21} \text { odd }\right) \\
\mathbf{B}_{r s}^{p 2} & \left(\alpha^{\prime}, \beta^{\prime}\right) \\
= & U_{r-1}\left(\frac{2}{a_{2 p}-a_{1 p}}\left[\alpha^{\prime}-\frac{a_{2 p}+a_{1 p}}{2}\right]\right) \\
& \times \sqrt{1-\left(\frac{2}{a_{2 p}-a_{1 p}}\left[\alpha^{\prime}-\frac{a_{2 p}+a_{1 p}}{2}\right]\right)^{2} \mathrm{e}\left[s-\left(\frac{N_{22}+1}{2}\right)\right] \beta^{\prime}} \\
& \quad \times\left(\eta \cos \beta^{\prime} \hat{\mathbf{x}}+\sin \beta^{\prime} \hat{\mathbf{y}}\right) \quad\left(a_{1 p} \leq \alpha^{\prime} \leq a_{2 p} ; 0 \leq \beta^{\prime}<2 \pi\right) \\
& \left(r=1, \ldots, N_{12} ; s=1, \ldots, N_{22} ; N_{22} \text { odd }\right) .
\end{aligned}
$$

In the case of the elliptical arc of Fig. 2(e), we will assume that $b_{2 p} / a_{2 p}=b_{1 p} / a_{1 p}=\eta<1$ as in the case of the elliptical ring of Fig. 2(c). Under that assumption, the edge singularity basis functions we have chosen for the elliptical arc are

$$
\begin{aligned}
& \mathbf{B}_{r s}^{p 1}\left(\alpha^{\prime}, \beta^{\prime}\right) \\
& =\frac{T_{r-1}\left(\frac{2}{a_{2 p}-a_{1 p}}\left[\alpha^{\prime}-\frac{a_{2 p}+a_{1 p}}{2}\right]\right)}{\sqrt{1-\left(\frac{2}{a_{2 p}-a_{1 p}}\left[\alpha^{\prime}-\frac{a_{2 p}+a_{1 p}}{2}\right]\right)^{2}}} \\
& \times U_{s-1}\left(\frac{2}{\beta_{2 p}-\beta_{1 p}}\left[\beta^{\prime}-\frac{\beta_{2 p}+\beta_{1 p}}{2}\right]\right) \\
& \times \sqrt{1-\left(\frac{2}{\beta_{2 p}-\beta_{1 p}}\left[\beta^{\prime}-\frac{\beta_{2 p}+\beta_{1 p}}{2}\right]\right)^{2}} \\
& \times\left(-\sin \beta^{\prime} \hat{\mathbf{x}}+\eta \cos \beta^{\prime} \hat{\mathbf{y}}\right) \quad\left(a_{1 p}<\alpha^{\prime}<a_{2 p} ; \beta_{1 p} \leq \beta^{\prime} \leq \beta_{2 p}\right) \\
& \left(r=1, \ldots, N_{11} ; s=1, \ldots, N_{21}\right) \\
& \mathbf{B}_{r s}^{p 2}\left(\alpha^{\prime}, \beta^{\prime}\right) \\
& =U_{r-1}\left(\frac{2}{a_{2 p}-a_{1 p}}\left[\alpha^{\prime}-\frac{a_{2 p}+a_{1 p}}{2}\right]\right) \\
& \times \sqrt{1-\left(\frac{2}{a_{2 p}-a_{1 p}}\left[\alpha^{\prime}-\frac{a_{2 p}+a_{1 p}}{2}\right]\right)^{2}} \\
& \times \frac{T_{s-1}\left(\frac{2}{\beta_{2 p}-\beta_{1 p}}\left[\beta^{\prime}-\frac{\beta_{2 p}+\beta_{1 p}}{2}\right]\right)}{\sqrt{1-\left(\frac{2}{\beta_{2 p}-\beta_{1 p}}\left[\beta^{\prime}-\frac{\beta_{2 p}+\beta_{1 p}}{2}\right]\right)^{2}}}\left(\eta \cos \beta^{\prime} \hat{\mathbf{x}}+\sin \beta^{\prime} \hat{\mathbf{y}}\right) \\
& \left(a_{1 p} \leq \alpha^{\prime} \leq a_{2 p} ; \quad \beta_{1 p}<\beta^{\prime}<\beta_{2 p}\right) \\
& \left(r=1, \ldots, N_{12} ; s=1, \ldots, N_{22}\right)
\end{aligned}
$$

where

$$
\begin{aligned}
& \beta_{1 p}=\arctan \left(\frac{\tan \varphi_{1 p}}{\eta}\right) \\
& \beta_{2 p}=\arctan \left(\frac{\tan \varphi_{2 p}}{\eta}\right),
\end{aligned}
$$

and where $\varphi_{1 p}$ and $\varphi_{2 p}$ are the angles defined in Fig. 2(e). The coordinates $\alpha^{\prime}$ and $\beta^{\prime}$ of (36) and (37) are the stretched polar coordinates defined in (32) and (33).

The basis functions for the circular arc of Fig. 2(d) can be obtained as a particular case of (36) and (37) when $\eta=1$, $a_{1 p}=\rho_{1 p}, a_{2 p}=\rho_{2 p}, \beta_{1 p}=\varphi_{1 p}, \beta_{2 p}=\varphi_{2 p}, \alpha^{\prime}=\rho^{\prime}$ and $\beta^{\prime}=\varphi^{\prime}$ (where $\rho^{\prime}$ and $\varphi^{\prime}$ are the polar coordinates defined in (28) and (29)).

In the case of the elliptical sector of Fig. 2(g), the basis functions proposed are

$$
\begin{aligned}
& \mathbf{B}_{r s}^{p 1}\left(\alpha^{\prime}, \beta^{\prime}\right) \\
& =\frac{T_{r-1}\left(\frac{\alpha^{\prime}}{a_{0 p}}\right)}{\sqrt{1-\left(\frac{\alpha^{\prime}}{a_{0 p}}\right)^{2}}\left(\frac{\alpha^{\prime}}{a_{0 p}}\right)^{\left[\left(\frac{\pi s}{\beta_{2 p}-\beta_{1 p}}\right)-1\right]}} \\
& \quad \times \sin \left[\frac{\pi s}{2}+\frac{\pi s}{\beta_{2 p}-\beta_{1 p}}\left(\beta^{\prime}-\frac{\beta_{2 p}+\beta_{1 p}}{2}\right)\right] \\
& \quad \times \sqrt{1-\left(\frac{2}{\beta_{2 p}-\beta_{1 p}}\left[\beta^{\prime}-\frac{\beta_{2 p}+\beta_{1 p}}{2}\right]\right)^{2}} \\
& \quad \times\left(-\sin \beta^{\prime} \hat{\mathbf{x}}+\eta \cos \beta^{\prime} \hat{\mathbf{y}}\right) \\
& \quad\left(0<\alpha^{\prime}<a_{0 p} ; \beta_{1 p} \leq \beta^{\prime} \leq \beta_{2 p}\right) \\
& \quad\left(r=1, \ldots, N_{11} ; s=1, \ldots, N_{21}\right)
\end{aligned}
$$




$$
\begin{aligned}
& \mathbf{B}_{r s}^{p 2}\left(\alpha^{\prime}, \beta^{\prime}\right) \\
& =U_{r-1}\left(\frac{\alpha^{\prime}}{a_{0 p}}\right) \sqrt{1-\left(\frac{\alpha^{\prime}}{a_{0 p}}\right)^{2}}\left(\frac{\alpha^{\prime}}{a_{0 p}}\right)^{\left[\left(\frac{\pi s}{\beta_{2 p}-\beta_{1 p}}\right)-1\right]} \\
& \quad \times \frac{\cos \left[\frac{\pi s}{2}+\frac{\pi s}{\beta_{2 p}-\beta_{1 p}}\left(\beta^{\prime}-\frac{\beta_{2 p}+\beta_{1 p}}{2}\right)\right]}{\sqrt{1-\left(\frac{2}{\beta_{2 p}-\beta_{1 p}}\left[\beta^{\prime}-\frac{\beta_{2 p}+\beta_{1 p}}{2}\right]\right)^{2}}} \\
& \quad \times\left(\eta \cos \beta^{\prime} \hat{\mathbf{x}}+\sin \beta^{\prime} \hat{\mathbf{y}}\right) \quad\left(0<\alpha^{\prime} \leq a_{0 p} ; \quad \beta_{1 p}<\beta^{\prime}<\beta_{2 p}\right) \\
& \quad\left(r=1, \ldots, N_{12} ; s=1, \ldots, N_{22}\right)
\end{aligned}
$$

where the coordinates $\alpha^{\prime}$ and $\beta^{\prime}$ have been defined in (32) and (33), and where $\beta_{1 p}$ and $\beta_{2 p}$ have been defined in (38) and (39).

As in the case of Fig. 2(d) in relation with (36) and (37), the basis functions for the circular sector of Fig. 2(f) can be obtained as a particular case of (40) and (41) when $\eta=1$, $a_{0 p}=\rho_{0 p}, \beta_{1 p}=\varphi_{1 p}, \beta_{2 p}=\varphi_{2 p}, \alpha^{\prime}=\rho^{\prime}$ and $\beta^{\prime}=\varphi^{\prime}$ ( $\rho^{\prime}$ and $\varphi^{\prime}$ are the polar coordinates defined in (28) and (29)). In the particular case of the circular sector, the factor $\left(\alpha^{\prime} / a_{0 p}\right)^{\left[\left((\pi s) /\left(\beta_{2 p}-\beta_{1 p}\right)\right)-1\right]}$ and the sinusoidal functions of $\left[(\pi s) /(2)+(\pi s) /\left(\beta_{2 p}-\beta_{1 p}\right) \times\left(\beta^{\prime}-\left(\beta_{2 p}+\beta_{1 p}\right) /(2)\right)\right]$ have been included in (40) and (41) to accommodate the behavior of the current density (electric and magnetic) far from the edges of the circular sector in accordance with the magnetic wall model of a microstrip circular sector proposed in [24], just as it was done in [13] for a circular microstrip patch.

\section{NUFFT of the Basis Functions}

Equations (7) and (8) show that the computation of the MoM matrix entries in the spectral domain requires the knowledge of the 2-D discrete Fourier transforms $\widetilde{\mathbf{J}}_{r s}^{p q, \mathrm{~d}}\left(k_{x m}, k_{y n}\right)$ and $\widetilde{\mathbf{M}}_{r s}^{p q, \mathrm{~d}}\left(k_{x m}, k_{y n}\right)$. The Fourier transforms of the edge singularity basis functions introduced in (24), (25), (30), (31), (34) to (37), (40) and (41) cannot be obtained in closedform, and this is an important drawback since the spectral domain MoM loses all its efficiency when the 2-D Fourier transforms of the basis functions are not available in closedform. In this subsection we show how the 2-D discrete Fourier transforms of all the edge singularity basis functions introduced in Subsection II-B can be numerically obtained in an efficient way by means of the NUFFT algorithm.

Let $F_{r s}^{p q}(x, y)$ represent any of the two components of $\mathbf{J}_{r s}^{p q}(x, y)$ and $\mathbf{M}_{r s}^{p q}(x, y)$. In the case of the basis functions for the patch/aperture of Fig. 2(a), the discrete Fourier transform of $F_{r s}^{p q}(x, y), \widetilde{F}_{r s}^{p q, \mathrm{~d}}\left(k_{x m}, k_{y n}\right)$, would be given by (see (10) and (11))

$$
\begin{aligned}
\widetilde{F}_{r S}^{p q, \mathrm{~d}}\left(k_{x m}, k_{y n}\right) & \\
= & \frac{\mathrm{e}^{-\mathrm{j}\left(k_{x 0} x_{0 \mathrm{c}}+k_{y 0} y_{\mathrm{oc}}\right)}}{a b} \int_{-L_{p}}^{+L_{p}} \\
\times & {\left[\int_{l_{p}^{-}\left(y^{\prime}\right)}^{l_{p}^{+}\left(y^{\prime}\right)} F_{r s}^{p q}\left(x^{\prime}, y^{\prime}\right) \times \mathrm{e}^{-\mathrm{j}\left(k_{x 0} x^{\prime}+k_{y 0} y^{\prime}\right)}\right.} \\
& \left.\times \mathrm{e}^{-\mathrm{j}\left\{m\left[\frac{2 \pi\left(x^{\prime}+x_{0 \mathrm{c}}\right)}{a}\right]+n\left[\frac{2 \pi\left(y^{\prime}+y_{0 \mathrm{c}}\right)}{b}\right]\right\}^{\prime}} d x^{\prime}\right] d y^{\prime} .
\end{aligned}
$$

In order to numerically carry out the integral of (42), we are going to sample the integrand in the values of $x^{\prime}$ and $y^{\prime}$ given by

$$
\begin{aligned}
y_{i p}^{\prime}= & -L_{p}+\frac{\Delta y_{p}^{\prime}}{2}+\Delta y_{p}^{\prime} i \quad\left(i=0, \ldots, N_{y}-1\right) \\
x_{i j, p}^{\prime}= & l_{p}^{-}\left(y_{i}^{\prime}\right)+\frac{\Delta x_{i p}^{\prime}}{2}+\Delta x_{i p}^{\prime} j \\
& \quad\left(i=0, \ldots, N_{y}-1 ; j=0, \ldots, N_{x}-1\right)
\end{aligned}
$$

where $\Delta y_{p}^{\prime}=2 L_{p} / N_{y}$ and $\Delta x_{i p}^{\prime}=2 l_{p}\left(y_{i p}^{\prime}\right) / N_{x}\left(l_{p}\left(y^{\prime}\right)\right.$ was defined in (27)). Then, the integral of (42) can be approximately computed by means of the equation

$$
\begin{aligned}
& \widetilde{F}_{r s}^{p q, \mathrm{~d}}\left(k_{x m}, k_{y n}\right) \approx \sum_{i=0}^{N_{y}-1} \sum_{j=0}^{N_{x}-1} \frac{\mathrm{e}^{-\mathrm{j}\left(k_{x 0} x_{\mathrm{oc}}+k_{y 0} y_{\mathrm{oc}}\right)}}{a b} \Delta x_{i p}^{\prime} \Delta y_{p}^{\prime} \\
& \times F_{r s}^{p q}\left(x_{i j, p}^{\prime}, y_{i p}^{\prime}\right) \mathrm{e}^{-\mathrm{j}\left(k_{x 0} x_{i j, p}^{\prime}+k_{y 0} y_{i p}^{\prime}\right)}
\end{aligned}
$$

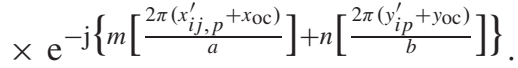

In practice, the numerical computation of $\Gamma_{k l, r s}^{i j, p q}$ and $\Lambda_{k l, r s}^{i j, p q}$ requires to truncate the infinite summations of (7) and (8) within the interval $-M / 2 \leq m, n<+M / 2$. For these particular values of $m$ and $n$, (45) can be rewritten as

$\widetilde{F}_{r s}^{p q, \mathrm{~d}}(m, n) \approx \sum_{k=0}^{N_{T}-1} \gamma_{k} \mathrm{e}^{-\mathrm{j}\left(m \mu_{k}+n v_{k}\right)} \quad(-M / 2 \leq m, n<+M / 2)$

where

$$
\begin{aligned}
\gamma_{k(i, j)}= & \frac{\mathrm{e}^{-\mathrm{j}\left(k_{x 0} x_{\mathrm{oc}}+k_{y 0} y_{\mathrm{oc}}\right)}}{a b} \Delta x_{i p}^{\prime} \Delta y_{p}^{\prime} F_{r s}^{p q}\left(x_{i j, p}^{\prime}, y_{i p}^{\prime}\right) \\
& \times \mathrm{e}^{-\mathrm{j}\left(k_{x 0} x_{i j, p}^{\prime}+k_{y 0} y_{i p}^{\prime}\right)} \quad\left(k=0, \ldots, N_{T}-1\right) \\
\mu_{k(i, j)}= & \frac{2 \pi\left(x_{i j, p}^{\prime}+x_{\mathrm{oc}}\right)}{a} \quad\left(k=0, \ldots, N_{T}-1\right) \\
v_{k(i, j)}= & \frac{2 \pi\left(y_{i p}^{\prime}+y_{\mathrm{oc}}\right)}{b} \quad\left(k=0, \ldots, N_{T}-1\right)
\end{aligned}
$$

and where $k(i, j)=i+N_{y} j\left(i=0, \ldots, N_{y}-1 ; j=0, \ldots\right.$, $\left.N_{x}-1\right)$, and $N_{T}=N_{x} N_{y}$.

The expression (46) is ready for the use of the NUFFT of type 1 as it appears in [17, Eqn. (1)], which can be efficiently implemented by means of the algorithm shown in [17, page 448]. A FORTRAN code for this algorithm is available at the internet link [25].

In the case of the patches/apertures of Fig. 2(b), (d), and (f), $\widetilde{F}_{r s}^{p q, \mathrm{~d}}\left(k_{x m}, k_{y n}\right)$ would be given by

$$
\begin{aligned}
& \widetilde{F}_{r s}^{p q, \mathrm{~d}}\left(k_{x m}, k_{y n}\right) \\
& =\frac{\mathrm{e}^{-\mathrm{j}\left(k_{x 0} x_{\mathrm{oc}}+k_{y 0} y_{\mathrm{oc}}\right)}}{a b} \int_{\rho_{\text {min }, p}^{\prime}}^{\rho_{\text {max }, p}^{\prime}} \\
& \times\left[\int_{\varphi_{\min , p}^{\prime}}^{\varphi_{\text {max }, p}^{\prime}} F_{r s}^{p q}\left(\rho^{\prime}, \varphi^{\prime}\right) \times \mathrm{e}^{-\mathrm{j}\left(k_{x 0} \rho^{\prime} \cos \varphi^{\prime}+k_{y 0} \rho^{\prime} \sin \varphi^{\prime}\right)}\right. \\
& \quad \times \mathrm{e}^{\left.-\mathrm{j}\left\{m\left[\frac{2 \pi\left(\rho^{\prime} \cos \varphi^{\prime}+x_{\mathrm{oc}}\right)}{a}\right]+n\left[\frac{2 \pi\left(\rho^{\prime} \sin \varphi^{\prime}+y_{\mathrm{oc}}\right)}{b}\right]\right\}^{\prime}\right] \rho^{\prime} d \rho^{\prime}}
\end{aligned}
$$


where $\left(\rho_{\min , p}^{\prime}, \rho_{\max , p}^{\prime}\right)=\left(\rho_{1 p}, \rho_{2 p}\right)$ in the case of Fig. 2(b) and $(\mathrm{d}),\left(\rho_{\min , p}^{\prime}, \rho_{\max , p}^{\prime}\right)=\left(0, \rho_{0 p}\right)$ in the case of Fig. $2(\mathrm{f})$, $\left(\varphi_{\min , p}^{\prime}, \varphi_{\max , p}^{\prime}\right)=(0,2 \pi)$ in the case of Fig. $2(\mathrm{~b})$, and $\left(\varphi_{\min , p}^{\prime}, \varphi_{\max , p}^{\prime}\right)=\left(\varphi_{1 p}, \varphi_{2 p}\right)$ in the case of Fig. 2(d) and (f).

If the variables $\rho^{\prime}$ and $\varphi^{\prime}$ of the integrand of (50) are sampled, it is possible to obtain an approximate expression for (50) of the type shown in (46). The expressions of $\gamma_{k}, \mu_{k}$ and $v_{k}$ would be in this case

$$
\begin{aligned}
\gamma_{k(i, j)}= & \frac{\mathrm{e}^{-\mathrm{j}\left(k_{x 0} x_{\mathrm{oc}}+k_{y 0} y_{\mathrm{oc}}\right)}}{a b} \rho_{i p}^{\prime} \Delta \rho_{p}^{\prime} \Delta \varphi_{p}^{\prime} F_{r s}^{p q}\left(\rho_{i p}^{\prime}, \varphi_{j p}^{\prime}\right) \\
& \times \mathrm{e}^{-\mathrm{j}\left(k_{x 0} \rho_{i p}^{\prime} \cos \varphi_{j p}^{\prime}+k_{y 0} \rho_{i p}^{\prime} \sin \varphi_{j p}^{\prime}\right)}\left(k=0, \ldots, N_{T}-1\right) \\
\mu_{k(i, j)}= & \frac{2 \pi\left(\rho_{i p}^{\prime} \cos \varphi_{j p}^{\prime}+x_{\mathrm{oc}}\right)}{a}\left(k=0, \ldots, N_{T}-1\right) \\
v_{k(i, j)}= & \frac{2 \pi\left(\rho_{i p}^{\prime} \sin \varphi_{j p}^{\prime}+y_{\mathrm{oc}}\right)}{b}\left(k=0, \ldots, N_{T}-1\right)
\end{aligned}
$$

where

$$
\begin{aligned}
& \rho_{i p}^{\prime}=\rho_{\text {min }, p}^{\prime}+\frac{\Delta \rho_{p}^{\prime}}{2}+\Delta \rho_{p}^{\prime} i \quad\left(i=0, \ldots, N_{\rho}-1\right) \\
& \varphi_{j p}^{\prime}=\varphi_{\min , p}^{\prime}+\frac{\Delta \varphi_{p}^{\prime}}{2}+\Delta \varphi_{p}^{\prime} j \quad\left(j=0, \ldots, N_{\varphi}-1\right)
\end{aligned}
$$

with $\Delta \rho_{p}^{\prime}=\left(\rho_{\max , p}^{\prime}-\rho_{\min , p}^{\prime}\right) / N_{\rho}, \Delta \varphi_{p}^{\prime}=\left(\varphi_{\max , p}^{\prime}-\right.$ $\left.\varphi_{\min , p}^{\prime}\right) / N_{\varphi}$, and $N_{T}=N_{\rho} N_{\varphi}$.

Finally, in the case of the patches/apertures of Fig. 2(c), (e) and (g), $\widetilde{F}_{r s}^{p q, \mathrm{~d}}\left(k_{x m}, k_{y n}\right)$ would be given by

$$
\begin{aligned}
& \widetilde{F}_{r s}^{p q, \mathrm{~d}}\left(k_{x m}, k_{y n}\right) \\
& =\frac{\mathrm{e}^{-\mathrm{j}\left(k_{x 0} x_{\mathrm{oc}}+k_{y 0} y_{\mathrm{oc}}\right)}}{a b} \int_{\alpha_{\min , p}^{\prime}}^{\alpha_{\max , p}^{\prime}} \\
& \times\left[\int_{\beta_{\min , p}^{\prime}}^{\beta_{\max , p}^{\prime}} F_{r s}^{p q}\left(\alpha^{\prime}, \beta^{\prime}\right) \mathrm{e}^{-\mathrm{j}\left(k_{x 0} \alpha^{\prime} \cos \beta^{\prime}+k_{y 0} \eta \alpha^{\prime} \sin \beta^{\prime}\right)}\right. \\
& \quad \times \mathrm{e}^{\left.-\mathrm{j}\left\{m\left[\frac{2 \pi\left(\alpha^{\prime} \cos \beta^{\prime}+x_{\mathrm{oc}}\right)}{a}\right]+n\left[\frac{2 \pi\left(\eta \alpha^{\prime} \sin \beta^{\prime}+y_{\mathrm{oc}}\right)}{b}\right]\right\}_{d \beta^{\prime}}\right]} \\
& \quad \times \eta \alpha^{\prime} d \alpha^{\prime}
\end{aligned}
$$

where $\left(\alpha_{\min , p}^{\prime}, \alpha_{\max , p}^{\prime}\right)=\left(a_{1 p}, a_{2 p}\right)$ in the case of Fig. 2(c) and (e), $\left(\alpha_{\min , p}^{\prime}, \alpha_{\max , p}^{\prime}\right)=\left(0, a_{0 p}\right)$ in the case of Fig. $2(\mathrm{~g})$, $\left(\beta_{\min , p}^{\prime}, \beta_{\max , p}^{\prime}\right)=(0,2 \pi)$ in the case of Fig. $2(\mathrm{c})$, and $\left(\beta_{\min , p}^{\prime}, \beta_{\max , p}^{\prime}\right)=\left(\beta_{1 p}, \beta_{2 p}\right)$ in the case of Fig. 2(e) and $(\mathrm{g})$.

If the variables $\alpha^{\prime}$ and $\beta^{\prime}$ of the integrand of (56) are sampled, it is possible to obtain an approximate expression of (56) in terms of these samples of the type shown in (46). In this particular case, the expressions for $\gamma_{k}, \mu_{k}$ and $\nu_{k}$ would be

$$
\begin{aligned}
\gamma_{k(i, j)}= & \frac{\mathrm{e}^{-\mathrm{j}\left(k_{x 0} x_{\mathrm{oc}}+k_{y 0} y_{\mathrm{oc}}\right)}}{a b} \eta \alpha_{i p}^{\prime} \Delta \alpha_{p}^{\prime} \Delta \beta_{p}^{\prime} F_{r s}^{p q}\left(\alpha_{i p}^{\prime}, \beta_{j p}^{\prime}\right) \\
& \times \mathrm{e}^{-\mathrm{j}\left(k_{x 0} \alpha_{i p}^{\prime} \cos \beta_{j p}^{\prime}+k_{y 0} \eta \alpha_{i p}^{\prime} \sin \beta_{j p}^{\prime}\right)}\left(k=0, \ldots, N_{T}-1\right) \\
\mu_{k(i, j)}= & \frac{2 \pi\left(\alpha_{i p}^{\prime} \cos \beta_{j p}^{\prime}+x_{\mathrm{oc}}\right)}{a}\left(k=0, \ldots, N_{T}-1\right) \\
v_{k(i, j)}= & \frac{2 \pi\left(\eta \alpha_{i p}^{\prime} \sin \beta_{j p}^{\prime}+y_{\mathrm{oc}}\right)}{b}\left(k=0, \ldots, N_{T}-1\right)
\end{aligned}
$$

where

$$
\begin{aligned}
& \alpha_{i p}^{\prime}=\alpha_{\min , p}+\frac{\Delta \alpha_{p}^{\prime}}{2}+\Delta \alpha_{p}^{\prime} i\left(i=0, \ldots, N_{\alpha}-1\right) \\
& \beta_{j p}^{\prime}=\beta_{\min , p}^{\prime}+\frac{\Delta \beta_{p}^{\prime}}{2}+\Delta \beta_{p}^{\prime} j\left(j=0, \ldots, N_{\beta}-1\right)
\end{aligned}
$$

with $\Delta \alpha_{p}^{\prime}=\left(\alpha_{\max , p}^{\prime}-\alpha_{\min , p}^{\prime}\right) / N_{\alpha}, \Delta \beta_{p}^{\prime}=\left(\beta_{\max , p}^{\prime}-\right.$ $\left.\beta_{\min , p}^{\prime}\right) / N_{\beta}$, and $N_{T}=N_{\alpha} N_{\beta}$.

\section{NumericAl RESUlts AND VALIDATIONS}

In all the results presented in this Section we have used $M=100$, which means that 10000 terms have been retained in the computation of the double infinite summations of (7) and (8). Also, the number of samples employed for an accurate determination of the NUFFT has been roughly $N_{T} \approx 10000$ (around 100 samples per coordinate). Finally, the number of basis functions used per patch/aperture for the convergence of the results ranges from $N_{b}=4$ to $N_{b}=18$. This implies that small MoM matrices have to be inverted, and therefore, clearly shows the advantage gained when using edge singularity basis functions in the application of the spectral domain MoM.

Fig. 3(a) shows results for the transmission through bandpass FSSs made of arrays of apertures with bow tie or barrel shape. In this case the transmission refers to the ratio between the total power transmitted and the incident power, even in the presence of grating lobes, which means that

$$
\text { Transmission }=\left.\sum_{m} \sum_{n} T_{m n}\right|_{k_{x m}^{2}+k_{y n}^{2}<k_{0}^{2}}
$$

In Fig. 3(a) the results obtained with the spectral domain MoM described in Section II are compared with the results published in [15, Fig. 4], and with results obtained with the commercial software CST. Excellent agreement is found among the three sets of results when four basis functions of the type shown in (24) and (25) are used in the approximation of the magnetic current density on the apertures. We can confirm that our MoM software is around 80 times faster than CST in a laptop computer with an Intel Core i7-4790 processor at 3.6 GHz, four cores and $32 \mathrm{~GB}$ of RAM. Fig. 3(b) shows the convergence of the results of Fig. 3(a) as we increase the number of basis functions used in the application of MoM. The results obtained with three basis functions are nearly coincident with those obtained for four basis functions, which indicates the fast convergence provided by the edge singularity basis functions.

Fig. 4(a) shows our spectral domain MoM-NUFFT results for the transmission of the fundamental Floquet harmonic $m=n=0$ through a bandpass FSS consisting of a periodic array of circular ring apertures. In the generation of these MoM-NUFFT results we have used the basis functions of (30) and (31) to approximate the magnetic current density on the rings. In Fig. 4(a) our MoM-NUFFT results are compared with the results plotted in [26, Fig. 2], and with results provided by CST. Excellent agreement is noticed between our results and CST results. Good agreement is found between our results and those of [26] for frequencies below the first Wood's anomaly (occurring for $(f b) / c=1$ ), but the results of [26] are 


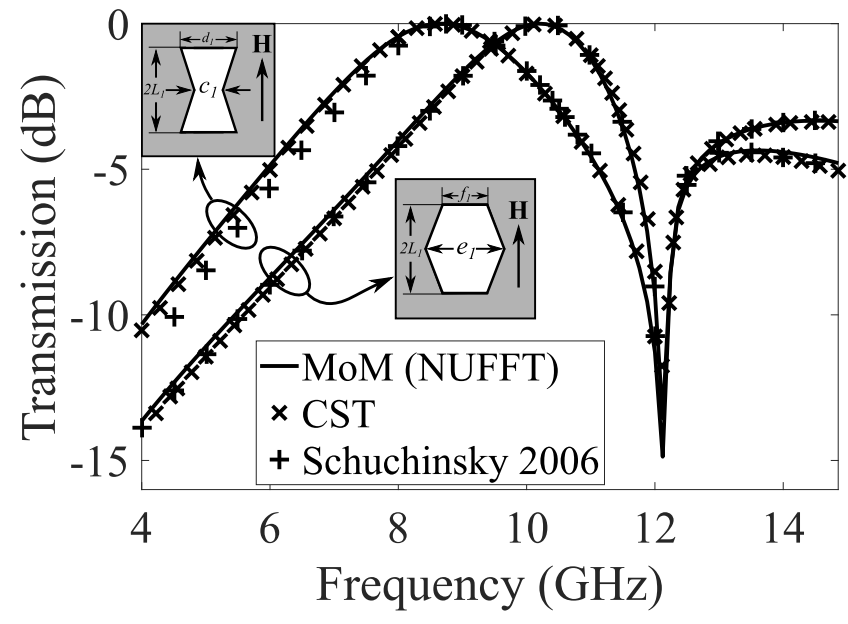

(a)

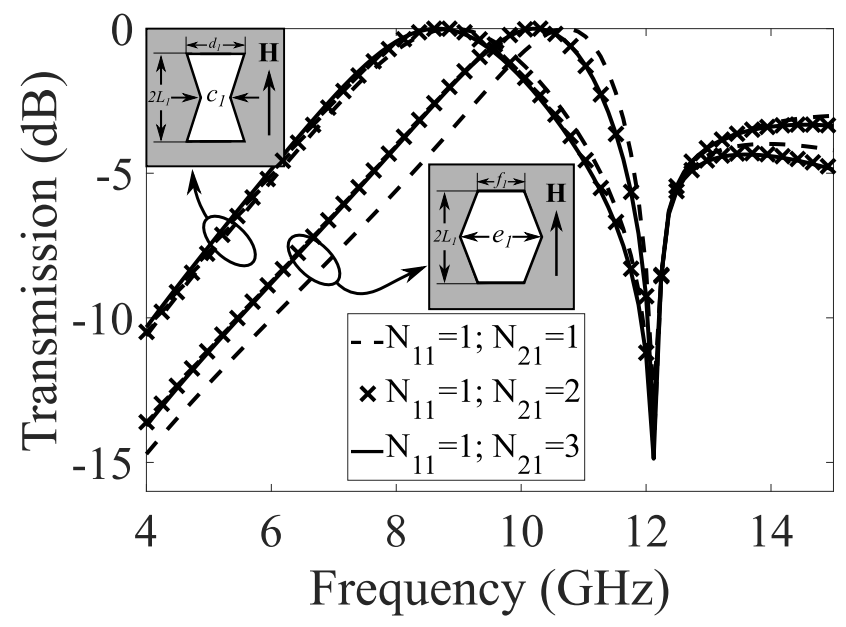

(b)

Fig. 3. (a) Transmission (in decibels) through two free-standing bandpass FSSs made of apertures with bow tie shape and barrel shape. Our results (solid lines) are compared with the results presented in [15, Fig. 4] (+), and with results provided by CST $(\times)$. (b) Convergence of the results shown in (a) with respect to the number of basis functions of the type shown in (24) when applying the spectral domain MoM with NUFFT. Parameters: $a=14.4 \mathrm{~mm}$; $b=17.26 \mathrm{~mm} ; 2 L_{1}=14.96 \mathrm{~mm} ; d_{1}=3 c_{1}=1.92 \mathrm{~mm}$ (bow-tie); $e=3 f_{1}=1.92 \mathrm{~mm}$ (barrel); $h_{1}=h_{2}=0 ; \theta_{\text {inc }}=45^{\circ} ; \varphi_{\text {inc }}=90^{\circ}$; magnetic field of the incident wave along the $y$-direction (TM polarization); $N_{11}=1$ and $N_{21}=3$ in (a); $N_{12}=N_{22}=1$ in (a) and (b).

far from the MoM-NUFFT results and the CST results when $(f b) / c>1.1$. This is to be expected since only one basis function without edge singularity was used in [26] to model the magnetic current density on the aperture (which justifies why the results of [26] do not match those of CST, even when $(f b) / c<1.1)$, and this single basis function is unable to approximate the variations of the magnetic current density when $(f b) / c>1.1$, i. e., at frequencies for which the ring is several wavelengths long. This is better explained in Fig. 4(b) where we plot the convergence of the transmission through the FSS with respect to the number of basis functions used in the approximation of the magnetic current density. Note that three basis functions suffice to provide excellent results for the transmission when $(f b) / c<1.1$, but this is not the case when $(f b) / c>1.1$, where the results obtained with just three basis

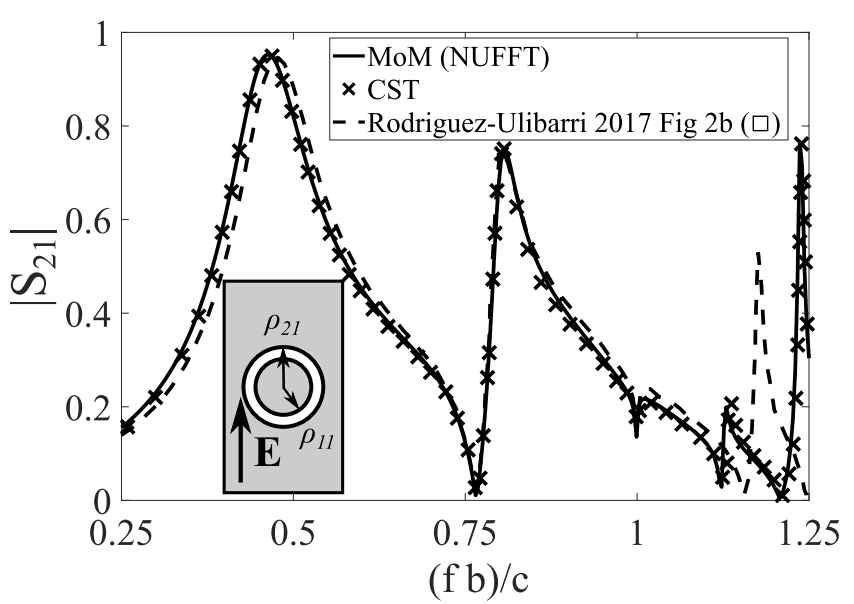

(a)

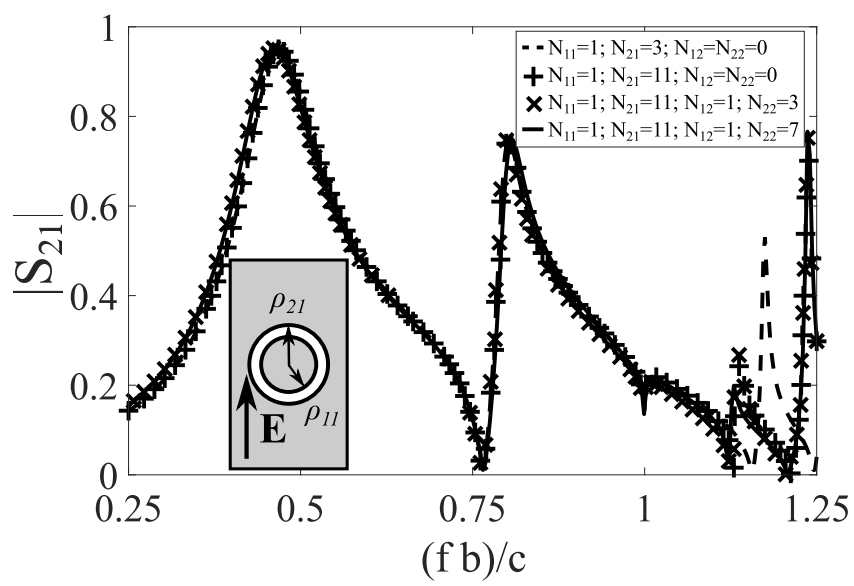

(b)

Fig. 4. (a) Transmission $\left(\left|S_{12}\right|=\sqrt{T_{00}}\right)$ in natural units through a bandpass FSSs made of an array of circular ring apertures on a dielectric substrate. Our results (solid line) are compared with the results presented in [26, Fig. 2(b), $\square$ ] (dashed line), and with results provided by CST $(\times)$. (b) Convergence of the results shown in (a) with respect to the number of basis functions of the type shown in (30) and (31) when applying the spectral domain MoM with NUFFT. Parameters: $a=1.5 \mathrm{~mm} ; b=3 \mathrm{~mm}$; $\rho_{11}=0.5 \mathrm{~mm} ; \rho_{21}=0.65 \mathrm{~mm} ; h_{1}=0.4 \mathrm{~mm} ; \varepsilon_{r, 1}=5 ; \tan \delta_{1}=0$; $h_{2}=0 \mathrm{~mm} ; \theta_{\mathrm{inc}}=0^{\circ}$; electric field of the incident wave along the $y$-direction; $N_{11}=1, N_{21}=11, N_{12}=1$ and $N_{22}=7$ in (a).

functions for the azimuthal component of the magnetic current density are not correct. Better results are obtained when the number of basis functions for the azimuthal component of the magnetic current density is increased up to 11 , but three additional basis functions for the radial component of the magnetic current density are required to achieve complete convergence. When 14 basis functions are used, we have noticed that our MoM software is around 50 times faster than CST in the laptop computer mentioned above.

In Fig. 5 we plot the phase of $R_{y y}$ (see (14)) for a linear polarization reflectarray element consisting of three parallel dipoles. Our MoM-NUFFT results are compared with CST results presented in [9, Fig. 3], and excellent agreement is found. The edge singularity basis functions of (24) and (25) have been used in the approximation of the electric current density on the dipoles, and we have found that four basis 


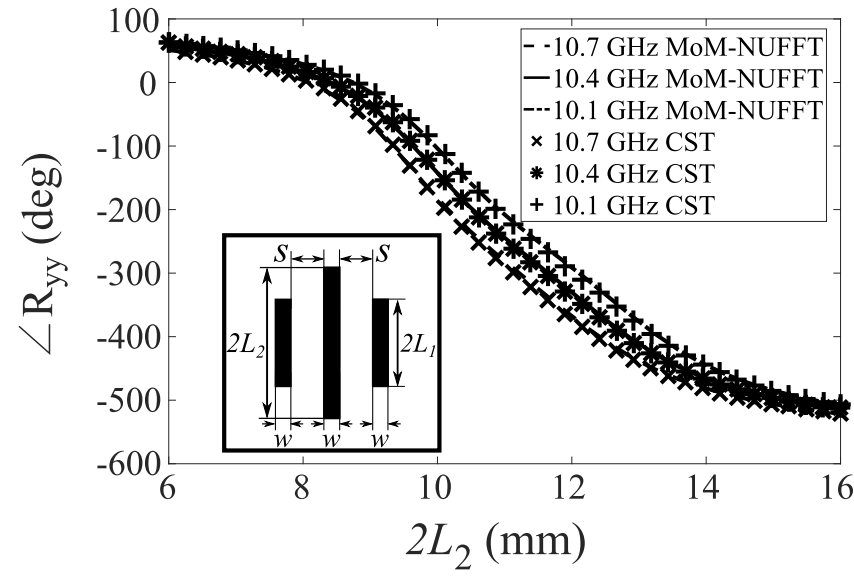

Fig. 5. Phase of $R_{y y}$ for a reflectarray element made of three parallel dipoles on a two-layered substrate. The phase is plotted as a function of the length of the longer dipole $2 L_{2}$. Our results (solid and dashed lines) are compared with the CST results shown in $[9$, Fig. 3] $(+, *, \times)$. Parameters: $a=b=16.5 \mathrm{~mm} ; L_{1}=0.7 L_{2} ; s=4.5 \mathrm{~mm} ; w=1 \mathrm{~mm} ; h_{1}=3 \mathrm{~mm}$ $\varepsilon_{r, 1}=1.067 ; \tan \delta_{1}=0.0002 ; h_{2}=0.508 \mathrm{~mm} ; \varepsilon_{r, 2}=3.38 ; \tan \delta_{2}=0.005$; $\theta_{\text {inc }}=0^{\circ} ; N_{11}=1, N_{21}=3, N_{12}=N_{22}=1$.

functions per dipole suffice to achieve convergent results in the values of $\angle R_{y y}$, which is in agreement with the results shown in the convergence plots of [9, Fig. 2(a)]. It turns out that for this particular case, the discrete 2-D Fourier transforms [see (10) and (11)] of the basis functions (24) and (25) can be obtained in closed-form in terms of Bessel functions [12]. So, we have implemented two codes for the spectral domain MoM of (1), (3), (5), (7) and (12), one in which the 2-D Fourier transforms are computed in closed form, and another one in which the 2-D Fourier transforms are computed by means of the NUFFT. When generating the results of Fig. 5, we have found that the code based on closedform Fourier transforms is only $15 \%$ faster than the code based on the NUFFT. In order to explain this small difference, we have to realize that the code based on closed-form Fourier transforms computes the Fourier transforms in a sequential way, and that the computation of all the Bessel functions needed in (7) is lengthy. However, the code based on the NUFFT provides all the required Fourier transforms of (7) in just one shot. So, the computational efficiency of the NUFFT algorithm counterbalances the mathematical efficiency introduced by the availability of analytical Fourier transforms. This statement implies that the spectral domain MoM-NUFFT code is competitive with the traditional spectral domain MoM of [2] in the cases where the Fourier transforms of the basis functions are available in closed-form.

In Fig. 6 the phases of $R_{\mathrm{RHCP}, \mathrm{RHCP}}$ and $R_{\mathrm{LHCP}, \mathrm{LHCP}}$ (see (15)) are plotted for a circular polarization reflectarray element consisting of two concentric split rings. These phases are used in [11] to design a dual-band circular polarization reflectarray. A comparison is carried out between our MoM-NUFFT results and the CST results published in [11, Fig. 7(b)], and excellent agreement is found. Our MoM-NUFFT results have been obtained with the circular arc version $(\eta=1)$ of the basis functions of (36) and (37) in the approximation of the electric current density on the arcs. Convergence results have been obtained with just four basis functions per circular arc, which is in agreement with the results shown in [11, Fig. 7(b)].

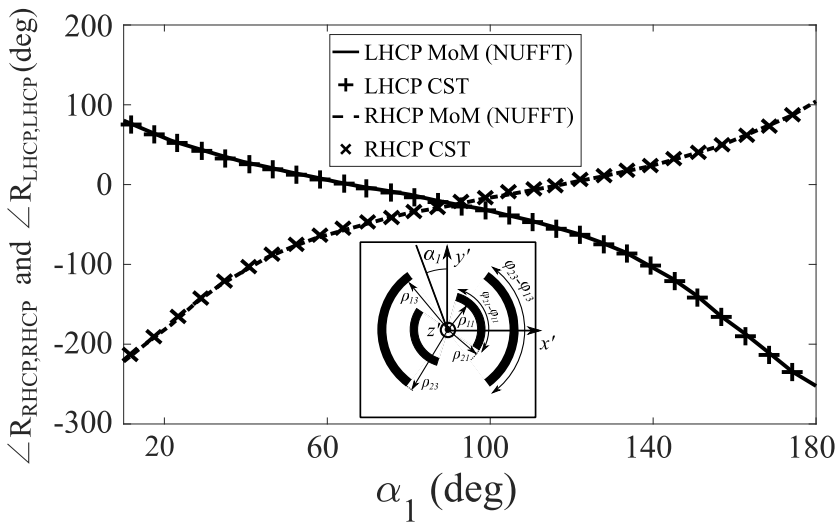

Fig. 6. Phase of $R_{\mathrm{RHCP}, \mathrm{RHCP}}$ and $R_{\mathrm{LHCP}, \mathrm{LHCP}}$ for a reflectarray element made of two concentric split rings on a one-layered substrate. The phase is plotted as the rotation angle of the inner ring $\alpha_{1}$. The plane $y^{\prime}-z^{\prime}$ is a mirror symmetry plane for the outer rings. Our results (solid and dashed lines) are compared with the CST results shown in [11, Fig. 7(b)] $(+, \times)$. Parameters: $a=b=5 \mathrm{~mm} ; \rho_{11}=\rho_{12}=1.2 \mathrm{~mm}$; $\rho_{21}=\rho_{22}=1.4 \mathrm{~mm} ; \rho_{13}=\rho_{14}=1.85 \mathrm{~mm} ; \rho_{23}=\rho_{24}=2.05 \mathrm{~mm}$; $\varphi_{21}-\varphi_{11}=\varphi_{22}-\varphi_{12}=\varphi_{23}-\varphi_{13}=\varphi_{24}-\varphi_{14}=150.4^{\circ} ;$ $h_{1}=0.787 \mathrm{~mm} ; \varepsilon_{r, 1}=2.2 ; \tan \delta_{1}=0.0009 ; h_{2}=0 \mathrm{~mm} ; \theta_{\text {inc }}=30^{\circ}$; $\varphi_{\text {inc }}=0^{\circ} ; f=29.75 \mathrm{GHz} ; N_{11}=1, N_{21}=3, N_{12}=N_{22}=1$.

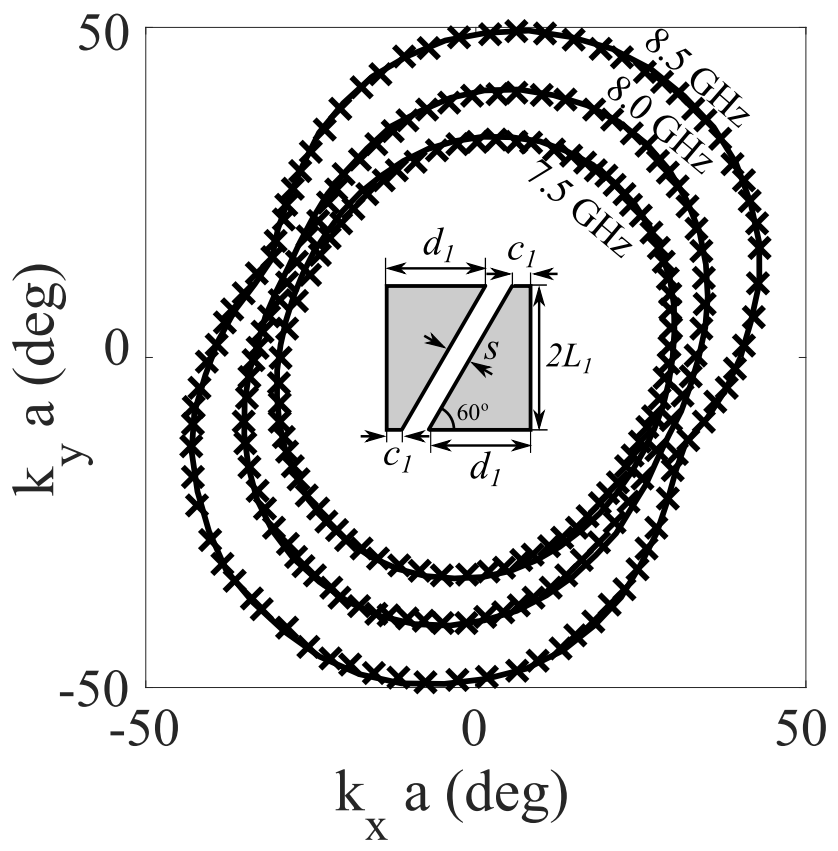

Fig. 7. Isofrequency dispersion contour for a periodic array of square patches with tilted slices on a one-layered substrate $\left(k_{x} a=k_{\rho \operatorname{sw}} \cos \alpha a\right.$ and $k_{y} a=k_{\rho \mathrm{sw}} \sin \alpha a$ ). Our results (solid lines) are compared with the full-wave simulations of [22, Fig. 7(b)] (×). Parameters: $a=b=3 \mathrm{~mm}$; $2 L_{1}=2.8 \mathrm{~mm} ; c_{1}=0.48 \mathrm{~mm} ; d_{1}=2.09 \mathrm{~mm} ; s=0.2 \mathrm{~mm} ; h_{1}=1.27 \mathrm{~mm}$; $\varepsilon_{r, 1}=10.2 ; \tan \delta_{1}=0 ; h_{2}=0 \mathrm{~mm} ; N_{11}=N_{21}=N_{12}=N_{22}=3$.

As an additional proof of the efficiency of the spectral domain MoM-NUFFT approach of Section II, we have compared the CPU time required by this approach to generate the results of Fig. 6 with the CPU time required by the Hankel transform approach introduced in [11] (which proved to be around 16 times faster than CST for the structure analyzed in Fig. 6, according to the results of [11, Table I]). The comparison indicates the MoM-NUFFT approach is around five times faster than the Hankel transform approach. Also, whereas the Hankel transform approach of [11] is especially tailored for the analysis of periodic structures in which the unit cell only 


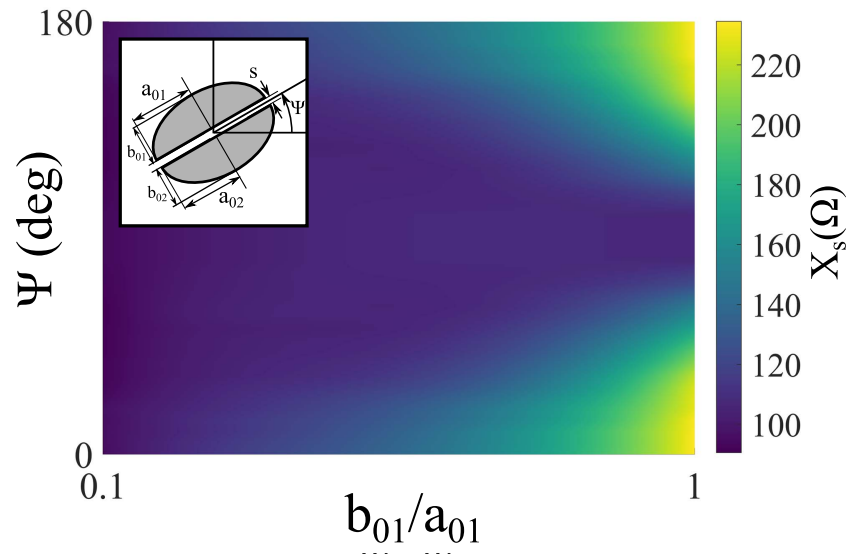

Fig. 8. Color map representing the equivalent impenetrable reactance $X_{S}$ of (20) for a periodic array of half-ellipses separated by a slot on a one-layered substrate. $X_{S}$ is represented as a function of the axial ratio of the ellipses and its rotation angle $\Psi$. The direction of wave propagation is $\alpha=0^{\circ}$. Parameters: $a=b=3 \mathrm{~mm} ; a_{01}=a_{02}=1.3 \mathrm{~mm} ; b_{01}=b_{02} ; s=0.2 \mathrm{~mm} ; h_{1}=3$ $\mathrm{mm} ; \varepsilon_{r, 1}=9.8 ; \tan \delta_{1}=0 ; h_{2}=0 \mathrm{~mm} ; f=11 \mathrm{GHz} ; N_{11}=2 ; N_{21}=3$; $N_{12}=N_{22}=1$.

contains concentric circular arcs, the MoM-NUFFT approach can be applied to a much wider variety of geometries as shown in Fig. 2.

Fig. 7 shows the isofrequency dispersion contours of a periodic array of square patches with tilted slices. The unit cell of this periodic structure was used in [27] to design a printed patch tensor holographic impedance surface. In Fig. 7 our MoM-NUFFT results are compared with the full-wave simulation results appearing in [22, Fig. 7]. Excellent agreement is found between both sets of results at all frequencies. The basis functions of (24) and (25) have been used to approximate the electric current density on each of the two trapezoids comprising the square patch with tilted slice. In order to obtain convergent results in the application of the MoM-NUFFT, 18 basis functions per trapezoid were used.

In Fig. 8 a color map is shown for the equivalent impenetrable reactance of a periodic array containting two half-elliptic patches separated by a slot in the unit cell. This is the scalar equivalent impenetrable reactance seen from the external side by a surface wave propagating along the positive $x$-direction of Fig. 1(b) [7]. In Fig. 8 the results for the reactance are plotted as a function of the axial ratio between the minor semi-axis and the major semi-axis of the half-ellipses, and as a function of the tilting angle of the slot. A similar element based on a circular patch with a tilted slice was used in [6] to design a circularly polarized MTS antenna. In fact, impedance color maps similar to that of Fig. 8 are shown in Fig. 12 of [6] where the impedance is plotted as a function of the radius of the sliced circular patches, and as a function of the tilting angle of the slices. Another similar MTS antenna element consisting of a tilted elliptic patch is studied in [7] where the impedance is plotted as a function of the ellipses axial ratio, and as a function of the ellipses tilting angle (see [7, Fig. 13]). The slotted elliptic patch studied in Fig. 8 can be viewed as a hybrid between the sliced circular patch of [6] and the elliptic patch of [7], and it is proposed here as an alternative element for the design of MTS antennas. In order to obtain the results of Fig. 8, the basis functions of (40) and (41) have been used in the approximation of the current density on the halfellipses where $\varphi_{21}-\varphi_{11}=180^{\circ}$ (i.e., the half ellipses have been treated as elliptic sectors). We have checked that seven basis functions per half-ellipse suffice to achieve convergence in the application of the spectral domain MoM-NUFFT. The generation of the results of Fig. 8 has required the computation of 20000 different MoM matrices, and this has required around $10 \mathrm{~h}$ of CPU time in the laptop computer mentioned above. This indicates the spectral domain MoM-NUFFT can be used as a powerful numerical tool for the design of MTS antennas.

\section{CONCLUSION}

In this paper the authors introduce an efficient spectral domain MoM approach for the analysis of a wide variety of multilayered periodic arrays of patches and apertures. The unit cell of these periodic arrays may contain either patches or apertures with the shape of a surface limited by two parallel lines and two arbitrary curves, with the shape of circular or elliptic rings, circular or elliptic arcs, and circular or elliptic sectors. Basis functions accounting for edge singularities are proposed for the approximation of the electric/magnetic current density on the patches/apertures of the periodic structures since these basis functions ensure a fast convergence of MoM with respect to the number of basis functions, and therefore, an efficient implementation of MoM. Since the 2-D Fourier transforms of these basis functions is not available in closed- form and is needed in the application of the spectral domain MoM, these 2-D Fourier transforms are numerically computed by means of the NUFFT algorithm in a fast and accurate way. The spectral domain MoM-NUFFT approach proposed is applied to the analysis of FSSs, and to the characterization of reflectarray antenna elements and MTS antenna elements. Comparison is carried out with results previously published in the literature and with results provided by commercial software CST, and good agreement is found in all cases. The combined use of the edge-singularity basis functions and the NUFFT in the application of the spectral domain MoM has made it possible to implement a code which is between 50 and 80 times faster than CST, and which is only $15 \%$ slower than the standard spectral domain MoM approach used for multilayered periodic structures in which the 2-D Fourier transform of the basis functions for the current density can be obtained in closed-form.

\section{ACKNOWLEDGMENT}

The authors would like to thank Dr. J. A. Encinar, Dr. M. Arrebola and Dr. R. Florencio for fruitful discussions on the use of the NUFFT for the characterization of reflectarray elements under the local periodicity assumption.

They would also like to thank Dr. Greengard and Dr. Lee for providing free internet access to their FORTRAN code on the NUFFT.

\section{REFERENCES}

[1] B. A. Munk, Frequency Selective Surfaces: Theory and Design. New York, NY, USA: Wiley, 2000.

[2] R. Mittra, C. H. Chan, and T. Cwik, "Techniques for analyzing frequency selective surfaces-A review," Proc. IEEE, vol. 76, no. 12, pp. 1593-1615, Dec. 1988. 
[3] J. Huang and J. A. Encinar, Reflectarray Antennas. New York, NY, USA: Wiley, 2008.

[4] D. M. Pozar, S. D. Targonski, and H. D. Syrigos, "Design of millimeter wave microstrip reflectarray," IEEE Trans. Antennas Propag., vol. 45, no. 2, pp. 287-296, Feb. 1997.

[5] G. Minatti, F. Caminita, M. Casaletti, and S. Maci, "Spiral leaky-wave antennas based on modulated surface impedance," IEEE Trans. Antennas Propag., vol. 59, no. 12, pp. 4436-4444, Dec. 2011.

[6] G. Minatti, S. Maci, P. De Vita, A. Freni, and M. Sabbadini, "A circularly-polarized isoflux antenna based on anisotropic metasurface," IEEE Trans. Antennas Propag., vol. 60, no. 11, pp. 4498-5009, Nov. 2012.

[7] M. Mencagli, E. Martini, and S. Maci, "Surface wave dispersion for anisotropic metasurfaces constituted by elliptical patches," IEEE Trans. Antennas Propag., vol. 63, no. 7, pp. 2992-3003, Jul. 2015.

[8] C. H. Chan and R. Mittra, "On the analysis of frequency-selective surfaces using subdomain basis functions," IEEE Trans. Antennas Propag. vol. 38 , no. 1, pp. 40-50, Jan. 1990.

[9] R. Florencio, R. R. Boix, E. Carrasco, J. A. Encinar, and V. Losada, "Efficient numerical tool for the analysis and design of reflectarrays based on cells with three parallel dipoles," Microw. Opt. Technol. Lett., vol. 55, no. 6, pp. 1212-1216, Jun. 2013.

[10] R. Florencio, R. R. Boix, J. A. Encinar, and G. Toso, "Optimized periodic MoM for the analysis and design of dual polarization multilayered reflectarray antennas made of dipoles," IEEE Trans. Antennas Propag. vol. 65 , no. 7, pp. 3623-3637, Jul. 2017.

[11] R. Florencio, R. R. Boix, and J. A. Encinar, "Efficient spectral domain MoM for the design of circularly polarized reflectarray antennas made of split rings," IEEE Trans. Antennas Propag., vol. 67, no. 3, pp. 1760-1771, Mar. 2019.

[12] A. M. Lerer and A. G. Schuchinsky, "Full-wave analysis of threedimensional planar structures," IEEE Trans. Microw. Theory Techn., vol. 41, no. 11, pp. 2002-2015, Nov. 1993.

[13] V. Losada, R. R. Boix, and M. Horno, "Resonant modes of circular microstrip patches in multilayered substrates," IEEE Trans. Microw. Theory Tech., vol. 47, no. 4, pp. 488-498, Apr. 1999.

[14] J.-T. Kuo, "Vector finite Hankel transform analysis of shielded single and coupled microstrip ring structures," IEEE Trans. Microw. Theory Techn., vol. 47, no. 11, pp. 2161-2164, Nov. 1999.

[15] A. G. Schuchinsky, D. E. Zelenchuk, A. M. Lerer, and R. Dickie, "Full-wave analysis of Layered aperture arrays," IEEE Trans. Antennas Propag., vol. 54, no. 2, pp. 490-502, Feb. 2006.

[16] A. Dutt and V. Rokhlin, "Fast Fourier transforms for nonequispaced data," SIAM J. Sci Comput., vol. 14, no. 6, pp. 1368-1393, 1993.

[17] L. Greengard and J.-Y. Lee, "Accelerating the nonuniform fast Fourier transform," SIAM Rev., vol. 46, no. 3, pp. 443-454, 2004

[18] F. L. Mesa, R. Marqués, and M. Horno, "A general algorithm for computing the bidimensional spectral Green's dyad in multilayered complex bianisotropic media: The equivalent boundary method," IEEE Trans. Microw. Theory Tech., vol. 39, no. 9, pp. 1640-1649, Sep. 1991.

[19] M. Camacho, R. R. Boix, and F. Medina, "Computationally efficient analysis of extraordinary optical transmission through infinite and truncated subwavelength hole arrays," Phys. Rev. E, Stat. Phys. Plasmas Fluids Relat. Interdiscip. Top., vol. 93, no. 6, 2016, Art. no. 063312

[20] R. Florencio, J. A. Encinar, R. R. Boix, V. Losada, and G. Toso, "Reflectarray antennas for dual polarization and broadband telecom satellite applications," IEEE Trans. Antennas Propag., vol. 63, no. 4, pp. 1234-1246, Apr. 2015.

[21] P. Baccarelli, S. Paulotto, and C. Di Nallo, "Full-wave analysis of bound and leaky modes propagating along 2D periodic printed structures with arbitrary metallisation in the unit cell," IET Microw. Antennas Propag., vol. 1, no. 1, pp. 217-225, Feb. 2007.

[22] A. M. Patel and A. Grbic, "Effective surface impedance of a printedcircuit tensor impedance surface (PCTIS)," IEEE Trans. Microw. Theory Tech., vol. 61, no. 4, pp. 1403-1413, Apr. 2013.

[23] G. Vecchi, P. Pirinoli, and M. Orefice, "On the use of cavity modes as basis functions in the full wave analysis of printed antennas," IEEE Trans. Antennas Propag., vol. 46, no. 4, pp. 589-594, Apr. 1998.

[24] W. F. Richards, J.-D. Ou, and S. A. Long, "A theoretical and experimental investigation of annular, annular sector, and circular sector microstrip antennas," IEEE Trans. Antennas Propag., vol. AP-32, no. 8 , pp. 864-867, Aug. 1984.

[25] L. Greengard and J.-Y. Lee. (Jan. 20, 2009). NUFFT (NFFT, USFFT) Software. Accessed: Feb. 22, 2009. [Online]. Available: https://cims.nyu.edu/cmcl/nufft/nufft.html
[26] P. Rodríguez-Ulibarri, M. Navarro-Cía, R. Rodríguez-Berral, F. Mesa, F. Medina, and M. Beruete, "Annular apertures in metallic screens as extraordinary transmission and frequency selective surface structures," IEEE Trans. Microw. Theory Tech., vol. 65, no. 12, pp. 4933-4946, Dec. 2017.

[27] B. H. Fong, J. S. Colburn, J. J. Ottusch, J. L. Visher, and D. F. Sievenpiper, "Scalar and tensor holographic artificial impedance surfaces," IEEE Trans. Antennas Propag., vol. 58, no. 10, pp. 3212-3221, Oct. 2010

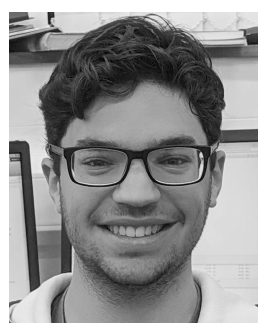

Miguel Camacho (S'17) was born in Seville, Spain, in 1993. He received the B.Sc. degree from the University of Seville, Seville, in 2015, and the Ph.D degree from the University of Exeter, Exeter, U.K., in 2019, all in physics.

$\mathrm{He}$ is currently a Post-Doctoral Researcher with the University of Pennsylvania, Philadelphia, PA, USA. He has authored ten peer-reviewed journal articles. He holds one patent. His current research interests include the numerical methods for the analysis of periodic and truncated arrays and metasurfaces.

Dr. Camacho received a Student Research Fellowship from the Spanish Ministry of Education and was selected to work as a summer student at the "Deutsches Elektronen-Synchrotron," Hamburg, Germany. He was a recipient of several awards from Seville's City Hall and the University of Seville for the Best Academic Record of the College of Physics from 2011 to 2015, the Spanish Ministry of Education National Award as the Best Physics Undergraduate, the IEEE Antennas and Propagation Society Doctoral Grant, and the Young Scientist Award of URSI Commission B in 2019.

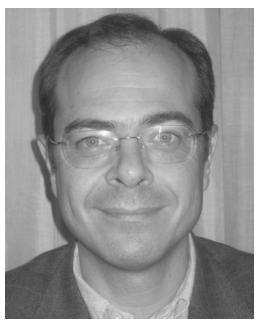

Rafael R. Boix (M'96) received the Licenciado and $\mathrm{Ph} . \mathrm{D}$. degrees in physics from the University of Seville, Seville, Spain, in 1985 and 1990, respectively.

Since 1986, he has been with the Electronics and Electromagnetism Department, University of Seville, where he became a Tenured Professor in 2010. His current research interests include the efficient numerical analysis of periodic planar multilayered structures with applications to the design of frequency selective surfaces, reflectarray antennas, metasurface antennas, and the development of circuit approaches for the synthesis of broadband microstrip antennas with frequency selective response.

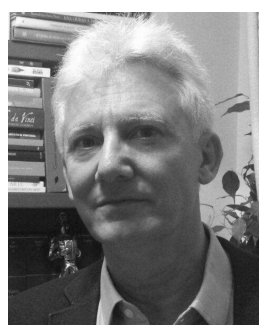

Francisco Medina (M'90-SM'01-F'10) was born in Cádiz, Spain, in 1960. He received the Licenciado and Ph.D. degrees in physics from the University of Seville, Seville, Spain, in 1983 and 1987, respectively.

$\mathrm{He}$ is currently a Professor of electromagnetism with the Department of Electronics and Electromagnetism, University of Seville, where he is also the Head of the Microwaves Group. His current research interests include analytical and numerical methods for the analysis of planar structures, anisotropic materials, artificial media modeling, and planar microwave circuits. He has authored or coauthored a number of book chapters, journal papers, and conference papers on these topics.

Dr. Medina is a member of the Editorial Board of the International Journal of RF and Microwave Computer-Aided Engineering. He is the Editor-in-Chief of the International Journal of Microwave and Wireless Technologies. 